\title{
Status quo der betriebswirtschaftlichen Erfolgsfaktorenforschung bei Büchern
}

\author{
Eine kritische Analyse der empirischen Literatur
}

\section{Eva Blömeke / Michel Clement / llaha Mahmudova / Frank Sambeth}

In diesem Aufsatz wird der Status quo der theoretischen und empirischen Erfolgsfaktorenforschung für belletristische Bücher aufgezeigt. Die abgeleiteten Einflussfaktoren, die zentrale Stenerungsgrößen für das Management der Verlagshäuser darstellen, werden nach den vier Elementen des Marketing-Mix-Produkt, Preis, Kommunikation und Distribution-untergliedert. Neben inhaltlichen Erkenntnissen stehen auch methodische Aspekte im Fokus, denn die gewählte Forschungsweise hat in der Erfolgsfaktorenforschung erbebliche inhaltliche Konsequenzen. Die Ergebnisse zeigen, dass sich die bisherige Faktorenforschung auf den Einfluss des Starwerts des Autors, die Aktualität des Inhaltes, die Editionsform und Cross-Selling (Komplementärgüter) sowie den Einfluss der Werbung für Kunden, Rezensionen durch Kritiker bzw. Kunden und Bestsellerlisten konzentriert. Somit liegt der Schwerpunkt der bisherigen Forschung auf Variablen der Produkt- und Kommunikationspolitik. Der Verkaufspreis als Kerndeterminante der Preispolitik findet ebenfalls Berücksichtigung, während im Bereich der Distributionspolitik nur der Einfluss von Verlagsgröße und Verlagsimage genauer untersucht wurde.

Schlagwörter: Buchmarktforschung, Erfolgsfaktorenforschung, Innovationsforschung, Marketing-Mix

\section{Einleitung}

Die betriebswirtschaftliche Erfolgsfaktorenforschung beschäftigt sich mit der Fragestellung, welchen Beitrag zentrale Steuergrößen des Managements auf den Erfolg eines Produktes haben. Hierbei geht es nicht nur darum festzustellen, ob ein Einfluss vorliegt, sondern auch wie hoch dieser im Vergleich $\mathrm{zu}$ anderen empirisch betrachteten Wirkungen ist. So ist unmittelbar einsichtig, dass der Autor eines Buches einen Einfluss auf den Erfolg hat - aber es ist nicht eindeutig festzulegen, ob diese Wirkung größer ist als z. B. die Werbewirkung oder der Preis des Buches. Auf Basis der Ergebnisse der empirischen Erfolgsfaktorenforschung kann das Unternehmen seine Maßnahmen insbesondere im Bereich des Marketings optimieren. Neben dem wirtschaftlichen Aspekt ist ebenfalls die wissenschaftliche Betrachtung der Erfolgsfaktorenforschung bei Medienprodukten von hoher Relevanz, denn aufgrund der besonderen Produkteigenschaften von hedonischen Gütern sind die Erkenntnisse aus anderen Industrien nicht ohne Weiteres auf Medienprodukte übertragbar. Sie erfordern daher eine dezidierte Analyse.

Entsprechend adressieren eine Vielzahl von Autoren die Erfolgsfaktoren von Medienprodukten wie Filme oder Musik (Eliashberg/Elberse/Leenders, 2006; Lee/Boatwright/Kamakura, 2003), so dass sich teilweise bereits empirische Generalisierungen ableiten lassen (Clement, 2004). Im Gegensatz zu der sehr umfangreichen empirischen Forschung bei Filmen hat die Erfolgsfaktorenforschung bei Büchern jedoch bei Weitem noch nicht das Niveau des Forschungsstands von Produktinnovationen im Allgemeinen erreicht (Hauschildt, 2004). Insgesamt kann die empirische Forschungslage hier als sehr disparat bezeichnet werden, da es keine einheitlichen Standards hinsichtlich der relevanten Grundgesamtheit, der verwendeten Methodik oder bezüglich der Erfolgs- 
maße und Einflussgrößen gibt. Dies führt dazu, dass die Vergleichbarkeit der Ergebnisse vorhandener Studien aus den Bereichen der Buchmarkt-, Lese- und Bestsellerforschung oftmals nur unzureichend gegeben ist.

Die bestehende Forschungslücke lässt sich mit der schwierigen Datenlage erklären. Während für Spielfilme Produktinformationen und Umsatzdaten öffentlich im Internet verfügbar sind (z. B. über IMDB - „The Internet Movie Database“), liegen diese Daten für Bücher nicht öffentlich vor und erschweren so die empirische Forschung. ${ }^{1}$ Zwar betreiben Verlage ebenfalls Untersuchungen zu den Erfolgstreibern ihrer Produkte, jedoch werden die Ergebnisse kaum publiziert, um einen möglichen Wettbewerbsvorsprung zu wahren.

In der Buchbranche wird jährlich ein Gesamtumsatzvolumen von knapp 9,3 Milliarden Euro allein in Deutschland erzielt (Börsenverein, 2007: 5). Aufgrund der hohen wirtschaftlichen Relevanz - und trotz der schlechten Datenlage - hat sich die theoretische und empirische Forschung mit dem Markt auseinandergesetzt. In den Forschungsbereichen der Wirtschafts-, Kommunikations- und Literaturwissenschaft sowie der Soziologie wurden mehrere empirische Studien zu den Erfolgsfaktoren von Büchern durchgeführt. Hierbei ist zumeist auf den Belletristikmarkt abgestellt worden, der mit etwa einem Drittel des Jahresumsatzes im Buchmarkt eine zentrale Stellung einnimmt und daher auch im Zentrum dieses Aufsatzes steht (Börsenverein, 2007: 56). Auch das Forschungsinteresse am Verlagsmanagement nimmt insbesondere in der MarketingDisziplin deutlich zu (Chevalier/Mayzlin, 2006; Clement/Proppe/Sambeth, 2006), so dass es an der Zeit ist, den bisherigen Wissensstand zur Erfolgsfaktorenforschung bei Büchern systematisch aufzubereiten und inhaltliche sowie methodische Forschungslücken zu kennzeichnen.

Das Buch als klassisches hedonisches Produkt ist ein Erfahrungsgut, bei dem der Wunsch nach Vergnügen und emotionaler Erregung im Vordergrund des Kaufinteresses steht (Dhar/Wertenbroch, 2000: 61; Hirschman/Holbrook, 1982: 92; Lageat/Czellar/Laurent, 2003: 98; Tietzel, 1995: 57). Wichtige Merkmale hedonischer Güter sind der innovative Charakter dieser Produkte, die unvollständige Substituierbarkeit und ein hohes Konsumrisiko. So hat der Kunde beim Kauf eines bestimmten Buches ein Informationsdefizit, und es fällt ihm schwer, das Produkt vor dem tatsächlichen Konsum zu bewerten. Die Informationsbeschaffung über die Lesewürdigkeit des Buches - z. B. durch Kritiker oder Bestsellerlisten (Clement/Proppe/Sambeth, 2006: 2ff.; Sorensen, 2004: 2ff.) - kann jedoch deutlich durch das Marketing beeinflusst werden.

Eine differenzierte Betrachtung der Marketinggesichtspunkte ist zentral für das Verlagsmanagement, denn der hohe Grad an Unsicherheit macht es aus Unternehmensperspektive schwierig, das Käuferverhalten abzuschätzen und die zu erwartende Gesamtnachfrage nach Büchern exakt zu prognostizieren. Dabei stellt jedes neue Buch für den Verlag eine Produktinnovation dar (Prosi, 1971: 21; Tietzel, 1995: 152). Ein Blick auf die gesamte Branchenentwicklung offenbart seit Jahren steigende Produktionszahlen auf dem internationalen Buchmarkt (Börsenverein, 2007: 58). Die Kenntnis der erfolgskritischen Faktoren würde die Entscheidung über knappe Ressourcen und die effiziente Allokation des Marketingbudgets erleichtern und somit die Wettbewerbsposition von Verlagen verbessern.

Der vorliegende Beitrag adressiert diese Problematik und vergleicht die bestehenden

1 In Deutschland (ebenso wie in den USA) gibt es keine institutionalisierte empirische Buchmarktforschung, wie dies beispielsweise für den Bereich Print oder TV der Fall ist (Bonfadelli, 2004; Clement, 2004). 
empirischen Studien, um so den Status quo der Erfolgsfaktorenforschung von Büchern aufzuzeigen. Dabei werden im Detail die Datenbasis, die eingesetzte Methodik sowie die Ergebnisse der verschiedenen Studien dargestellt und diskutiert. Der Beitrag verfolgt demnach zwei zentrale Ziele:

- Inhaltliches Ziel: Es wird der Status quo der empirischen Erfolgsfaktorenforschung für belletristische Bücher aufgezeigt. Neben einer systematischen Präsentation der Erkenntnisse wird die Relevanz der einzelnen Faktoren diskutiert.

- Methodisches Ziel: Neben inhaltlichen Erkenntnissen stehen auch methodische Aspekte im Vordergrund. Die jeweils eingesetzte Methodik zur Analyse der Erfolgsfaktoren der Studien wird im Detail dargelegt, da die gewählte Forschungsweise in der Erfolgsfaktorenforschung teilweise zu erheblichen inhaltlichen Konsequenzen führt.

Als Ergebnis sollen sowohl Implikationen inhaltlicher Art, d. h. für das Management, als auch methodischer Art und damit die Forschung adressierend abgeleitet werden. Der Beitrag ist wie folgt gegliedert: Im zweiten Abschnitt wird kurz die Struktur des deutschen Buchmarktes dargestellt. Danach schließt sich die Übersicht der bisherigen theoretischen und empirischen Erfolgsfaktorenforschung im Buchbereich an. Dabei wird zunächst ein Literaturüberblick gegeben, um im Anschluss die identifizierten Faktoren im Detail zu diskutieren. Der Beitrag endet mit einer Zusammenfassung der zentralen Ergebnisse und den Implikationen für die weitere Forschung.

\section{Der Buchmarkt in Deutschland}

Die Struktur des Buchmarktes lässt sich durch die Darstellung der Angebots- und Nachfrageseite erläutern. Das Angebot, mit dem die Konsumenten auf dem Buchmarkt konfrontiert werden, wird vor allem durch die Verlagshäuser bestimmt. Auf Basis der vorliegenden Manuskripte in- und ausländischer Autoren werden einzelne Buchtitel für die Herausgabe ausgewählt (Hjorth-Andersen, 2000: 28f.). Die Auswahl des Manuskripts ist zentral für den späteren Erfolg des Buches, so dass Verleger bereits in dieser frühen Wertschöpfungsstufe nach kritischen Erfolgsfaktoren suchen. Diese erste Stufe im Rahmen der Produktpolitik ist umso wichtiger, als dass beispielsweise die Preispolitik stark durch das Buchpreisbindungsgesetz reglementiert wird (van der Ploeg, 2004: 2ff.).

Die deutsche Buchbranche erzielt ein Gesamtumsatzvolumen von rund 9,3 Milliarden Euro (Börsenverein, 2007: 5). Etwa ein Drittel des Jahresumsatzes im Buchmarkt wird durch den Bereich Belletristik bestimmt (Börsenverein, 2007: 9f.). Es folgen die Warengruppen „Sachbuch und Ratgeber“ mit etwa 18\% sowie die „Kinder- und Jugendliteratur" mit 13\% Umsatzanteil. Auch wenn durch den Verkauf von HardcoverBüchern insgesamt über $70 \%$ des Gesamtumsatzes generiert werden, sind Taschenbücher besonders im Belletristikmarkt relevant und die umsatzbezogene Verteilung der beiden Editionsformen ist hier nahezu gleich gewichtet. Interessant ist die Entwicklung im Genre der Kinder- und Jugendbuchliteratur. So ließ sich in den letzten Jahren beobachten, dass die „Harry-Potter"-Serie einen deutlichen Einfluss in dieser Kategorie hat. Entsprechend der Neuerscheinungen kann zwischen den Jahren 2003 bis 2006 ein Zickzackkurs mit Umsatzschwankungen um zwei Prozent festgestellt werden (Börsenverein, 2007: 9ff.).

Seit Jahren zeichnet sich auf dem deutschen Buchmarkt der Trend steigender Buchproduktionszahlen ab. Die Produktion der deutschen Verlagshäuser stieg noch im Jahr 2005 um knapp 2\% gegenüber dem Vorjahr an (Börsenverein, 2006a: 53). Erst im letzten Jahr ging die Zahl der produzierten Bücher erstmals um ein Prozent zurück. Der Wert 
pro hergestelltem Exemplar lag 2006 im Durchschnitt bei 4,57 Euro. Insbesondere im letzten Jahr zeigt sich sehr deutlich der Trend zum Rückgang der Durchschnittspreise, was u. a. auch auf das Erscheinen der Bestsellerbibliotheken vieler Zeitungshäuser zurückzuführen ist (Börsenverein, 2007: 48ff.).

\section{Abbildung 1: Neuerscheinungen der Jahre 1995-2006 (Börsenverein, 2007: 57f.)}

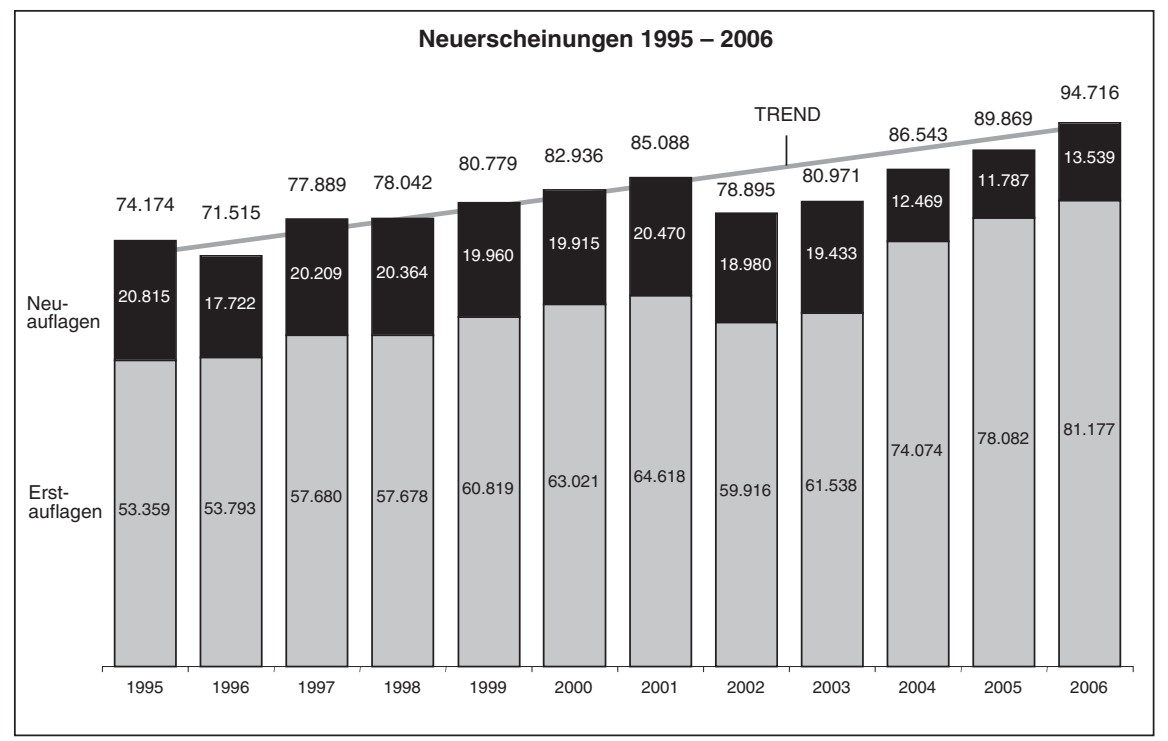

Die Anzahl der Neuerscheinungen der deutschen Verlage zeigt eine positive Tendenz. Nach einem kurzfristigen Rückgang 2002 wurde 2006 mit 94.716 Titeln ein neuer Rekord erreicht (siehe Abbildung 1). Dies bedeutet gegenüber dem Vorjahr eine Steigerung um knapp 5\%. Anhand der Grafik ist zu erkennen, dass das erzielte Wachstum hauptsächlich im Bereich der neu erschienenen Buchtitel (Erstauflagen) entstanden ist. Ihre Anzahl stieg im Jahr 2006 um mehr als vier Prozent (Börsenverein, 2007: 57f.). ${ }^{2}$

Die steigende Titelproduktion wird branchenintern seit langem kritisiert, da sie die Branche wirtschaftlich belastet. Die Herausgabe jedes neuen Buches ist mit Kosten und Risiken verbunden und nur wenige Buchtitel werden zu Bestsellern. Die Budgetlimitation der Verlage erfordert eine Fokussierung auf bestimmte Titel zur gezielten Absatzförderung, denn erst Bücher mit einer verkauften Auflage von etwa 5.000 Stück zählen zu den „erfolgreichen Büchern“ (Baumgarth, 2004: 49f.). Eine optimale Verteilung des Budgets ist jedoch nur möglich, wenn dem Verlag die Wirkung der einzelnen Marketinginstrumente bekannt ist.

Sammeleditionen der großen Zeitungshäuser, die so genannten „Billig-Bücher“, stellen Produktinnovationen auf dem Buchmarkt dar, die von vielen Verlegern als bedrohlich empfunden wurden. Es ist jedoch bereits ein Trend zur Konsolidierung in diesem Segment zu erkennen, denn im Vergleich zum Vorjahr konnte im Jahr 2005 kein zusätz-

2 Eine leichte Verzerrung ist durch Veröffentlichungen von Institutionen außerhalb der klassischen Branchenunternehmen gegeben, die erst seit dem Jahr 2004 in dieser Statistik berücksichtigt werden. 
liches Umsatzwachstum generiert werden. Das Marktsegment verteilt sich zunehmend auf mehrere Marktteilnehmer, wobei die „SZ-Bibliothek“ und die „Bild-Bestseller-Bibliothek" hier nach wie vor mit einem Marktanteil von 32\% (bzw. 36\%) dominieren (Börsenverein, 2006b: 5f.).

Neben öffentlichen Einrichtungen, wie Bibliotheken oder Universitäten, sieht sich der Handel - anders als die Verlage - auf Nachfragerseite insbesondere dem Privatkunden als Abnehmer gegenüber. Das Kaufverhalten auf dem Buchmarkt wird hier von kurz- und langfristigen Größen determiniert. $\mathrm{Zu}$ den langfristigen Faktoren gehören die Lesefähigkeit, der Ausbildungsgrad sowie die individuelle Freizeitgestaltung und die Nutzung von Medien (Zeitungen, Internet, Fernsehen etc.), die zum Buchlesen in Konkurrenz stehen (Hjorth-Andersen, 2000: 32f.). Unter den beliebtesten Freizeitbeschäftigungen nimmt das Bücherlesen Rang 6 von 44 Aktivitäten ein (Börsenverein, 2007: 15f.). Auch im Hinblick auf die Nutzungsdauer konnte das Buch seine Position in den letzten Jahren ausbauen. Im Durchschnitt liest der Deutsche (über 14 Jahre) 25 Minuten am Tag ein Buch (van Eimeren/Ridder, 2005: 499). Im Vergleich dazu verbringt er fast das 9-Fache mit der Nutzung von Radio oder Fernsehen (je 220 Minuten).

Saisonale Effekte (z. B. durch Feiertage) wirken sich ebenfalls auf die Nachfrage auf dem Buchmarkt aus. Insbesondere vor Weihnachten ist ein deutlicher Anstieg der Absatzzahlen zu beobachten (Beck, 2007: 3). Zu den kurzfristigen Faktoren, die das Buchkaufverhalten beeinflussen, gehören außerdem die einzelnen Buchtitel mit allen relevanten Attributen wie Inhalt, Ausstattung etc., die realen Buchpreise sowie Werbung.

Insgesamt haben 55\% der Deutschen (älter als 10 Jahre) im Jahr 2004 mindestens ein Buch gekauft (Börsenverein, 2005b: 5ff.). Die Gruppe der Buchkäufer lässt sich in drei Segmente unterteilen: Wenigkäufer (35\%) mit bis zu 7 Buchkäufen im Jahr, Durchschnittskäufer (10\%) mit 8-14 Büchern und Vielkäufer (10\%) mit mindestens 15 Büchern. Die Ergebnisse machen aber auch deutlich, dass fast die Hälfte aller Deutschen (45\%) überhaupt kein Buch gekauft hat. In diesem Fall muss es andere Formen der Beschaffung (Geschenk, Leihe etc.) oder einen sehr zeitversetzten Konsum geben, da nur 9\% der gleichen Kohorte im Vergleichszeitraum kein Buch gelesen haben. Die Zahlen decken eine grundsätzliche strukturelle Besonderheit des Buchmarktes auf und geben damit erste Hinweise auf mögliche Handlungsempfehlungen für das Verlagsmanagement. Käufer und Konsument des Produkts sind nicht immer identisch - was insbesondere bei der Kommunikationspolitik beachtet werden muss - und der Kauf (Adoption) führt nicht zwangsläufig zur unmittelbaren Nutzung (Akzeptanz) des Buches. Insofern ist abzuleiten, dass sich die Erfolgsfaktorenforschung sehr intensiv mit Nutzersegmenten auseinander setzen muss und die Konsumenten-Heterogenität mittels geeigneter statistischer Instrumente berücksichtigt werden sollte (z. B. durch Einsatz von Latent-ClassModellen, Clement/Proppe/Sambeth, 2006; Wedel/Kamakura, 2001).

Ähnliche Empfehlungen lassen sich aus den Altersstrukturen ableiten. Wird beispielsweise der Bereich Belletristik betrachtet, zeigt sich, dass nur 20\% der Belletristikkäufer jünger als 30 Jahre sind. Mit 40\% stellt die Altergruppe zwischen 30 und 49 Jahren den Hauptanteil an Belletristikkäufern dar (Börsenverein, 2005c: 8). Dies gilt es bei der Thematik und Positionierung von Neuerscheinungen zu berücksichtigen, denn um erfolgreich zu sein, müssen gezielt die Wünsche und Bedürfnisse der speziellen Zielgruppe angesprochen werden.

Die hohe Komplexität auf dem deutschen Buchmarkt ist dadurch gegeben, dass branchenweit gewisse Strukturen und Trends zu erkennen sind, eine dezidierte Analyse der einzelnen Warengruppen jedoch zu ganz unterschiedlichen Ergebnissen führen kann. Die Marktgegebenheiten innerhalb der Buchbranche zeigen damit Besonderheiten auf, 
so dass die Ergebnisse der Erfolgsfaktorenforschung aus anderen Medienbereichen nicht unmittelbar auf den Buchmarkt zu transferieren sind und eine detaillierte, gesonderte Betrachtung erforderlich ist.

\section{Theoretische und empirische Erfolgsfaktorenforschung bei Büchern}

Wie eingangs angedeutet, gibt es für den Buchmarkt keine einheitliche empirische Forschungsrichtung und es werden in den verschiedenen wissenschaftlichen Disziplinen differierende Untersuchungsdesigns eingesetzt (Bonfadelli, 2004: 63; Dijkstra, 1994: 5f.). Die betriebswirtschaftliche Forschung fokussiert sich auf die ökonomische Quantifizierung des Bucherfolgs. Wenngleich der Schwerpunkt der vorliegenden Literaturübersicht auf den wirtschaftswissenschaftlichen Studien liegt, werden auch die relevanten ökonomischen Ergebnisse aus angrenzenden Bereichen präsentiert, um ein möglichst umfassendes Bild der untersuchten Einflussfaktoren zu liefern.

Es lassen sich zwei wesentliche Forschungsrichtungen identifizieren (Abbildung 2). Einerseits werden in den Studien ökonomische Erfolgsmaße (z. B. Absatz) verwendet, wobei hier mittels Absatzreaktionsfunktionen der Einfluss der Erfolgsfaktoren auf Buchebene statistisch gemessen wird (i. d. R. anhand von Regressionsanalysen). Andererseits gibt es eine Vielzahl von Studien, die die Einstellung bzw. Kaufverhaltensabsicht, das Interesse oder die Präferenz hinsichtlich bestimmter Bücher zu erklären versuchen. Diese Untersuchungen können dem Bereich der Verhaltens- und Präferenzanalyse auf individueller Ebene zugeordnet werden.

\section{Abbildung 2: Überblick Erfolgsfaktorenforschung}

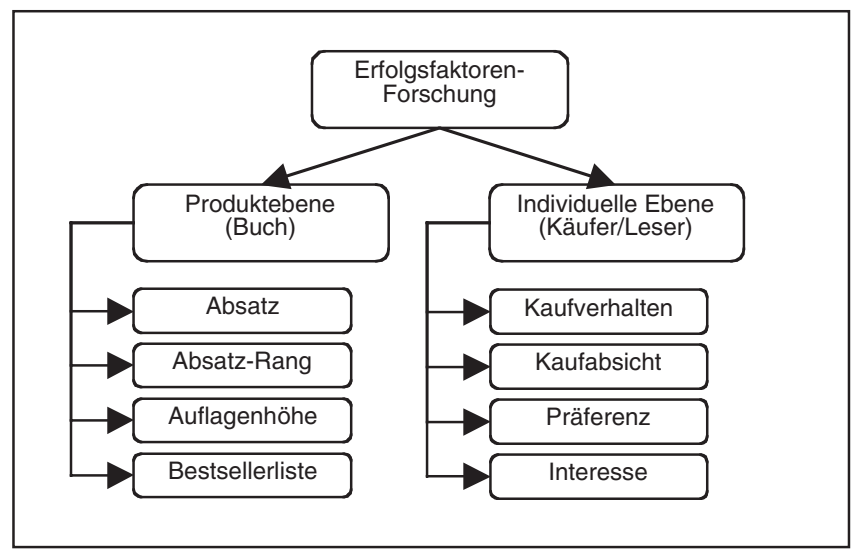

Die bestehende Literatur zur Lese-, Buchmarkt- und Bestsellerforschung lässt Erfolgsfaktoren ableiten, die teils theoretisch, teils empirisch gewonnen wurden. Um die Literaturanalyse sinnvoll abzugrenzen, wurde der Fokus auf die veröffentlichten Studien der letzten 20 Jahre gelegt. Innerhalb dieser Auswahl werden aufgrund der Aktualität die Ergebnisse seit dem Jahr 2000 verstärkt berücksichtigt. Darüber hinaus erfolgte eine Beschränkung auf Untersuchungen aus dem deutsch- und englischsprachigen Wissenschaftsraum. Tabelle 1 gibt zunächst einen detaillierten Überblick über die relevanten 
Informationen der untersuchten Studien zum ersten Teilbereich: der Erfolgsfaktorenforschung auf Buchebene. Die Studien sind nach dem Erfolgsmaß (Absatz, Bestsellerliste bzw. Auflagenhöhe) und anschließend nach Erscheinungsjahr gegliedert.

Um den Einfluss bestimmter Variablen auf den Buchabsatz zu überprüfen, wird generell auf die Regressionsanalyse als Analyseinstrument zurückgegriffen, wobei die ökonometrische Modellierung zunehmend an Qualität gewinnt, so dass auch die unbeobachtete Heterogenität nunmehr stärker kontrolliert wird (z. B. mittels Mixture Regression). Zusätzlich werden qualitative Auswertungen im Sinne einer Fallstudienanalyse durchgeführt. Eine Messung des Erfolgs anhand des Deckungsbeitrags wurde in keiner empirischen Untersuchung vorgenommen, so dass Aussagen bezüglich der Gewinnwirkung offenbleiben. Die Operationalisierung des Bucherfolgs erfolgt zumeist über den Absatz oder den Absatz-Rang. Chevalier/Goolsbee (2003) zeigen die Anpassung des Absatz-Rangs an den tatsächlichen Absatz, so dass das Maß als adäquate Alternative zum Absatz gilt. Die Verwendung von Rankings in Form von Bestsellerlisten hat den Vorteil, dass die Daten öffentlich verfügbar sind (Clement/Proppe/Sambeth, 2006: 802). Nachteilig ist jedoch die Konzentration auf bereits erfolgreiche Bücher.

Die Forschungsarbeiten aus dem Bereich der Verhaltens- und Präferenzanalyse befassen sich mit individuellen Kundendaten (Tabelle 2). ${ }^{3}$ Im Rahmen dessen gibt es zwei unterschiedliche Ansätze. Einerseits versucht man aus einer Stichprobe von Buchlesern oder -käufern Informationen mittels Befragung zu erheben. Hier erfolgt die Auswertung in der Regel durch Varianzanalysen (bzw. vorgeschaltete Faktorenanalysen). Darüber hinaus werden Experimente eingesetzt, bei denen die Probanden in simulierten Kaufsituationen beobachtet und anschließend befragt werden. Als abhängige Variable wird hier die Kaufabsicht, das Interesse oder die Präferenz für ein bestimmtes Buch zugrunde gelegt. Wenngleich die in Tabelle 2 dargestellten Untersuchungen gut geeignet sind, um beispielsweise explorativ neue Zusammenhänge aufzudecken, muss jedoch auf eine grundlegende Problematik hingewiesen werden. Durch das experimentelle Setting oder die Befragungs- bzw. Beobachtungssituation behält die Untersuchung immer einen hypothetischen Charakter. Dass die Konsumenteneinstellung und -absicht in vielen Fällen deutlich von der tatsächlichen Handlung abweicht, konnte empirisch bereits mehrfach bestätigt werden (z. B. Chandon/Morwitz/Reinartz, 2005). Um diesem Problem zu begegnen, untersuchen einige Studien bereits tatsächlich getätigte Buchkäufe. Hierbei handelt es sich jedoch um eine ex post Betrachtung, die weitere Risiken aufwirft (bereits verblasste Erinnerung, sozial erwünschte Antworten, Befragte geben ihrem Verhalten ex post einen rationalen Charakter, um kognitive Konsonanz herzustellen etc.). Sie liefern jedoch wichtige Hinweise - insbesondere direkt aus Kundenperspektive - auf mögliche Wirkungszusammenhänge, deren Kausalstruktur im Anschluss durch weitere Analysen überprüft werden muss.

3 Da der Fokus des vorliegenden Aufsatzes auf der ökonomischen Quantifizierung des Bucherfolgs liegt, wird für den zweiten Forschungsblock nur ein Ausschnitt präsentiert. Insbesondere auf zeitlich weit zurückliegende Studien soll an dieser Stelle nicht näher eingegangen werden (vgl. dazu z. B.: Harmgarth, 1997 und 1999; Köcher, 1988 und 1993; Martin/Scheer, 1995; Noelle-Neumann/Schulz, 1987; Schweitzer, 1997; Zentes/Effen, 1995). 


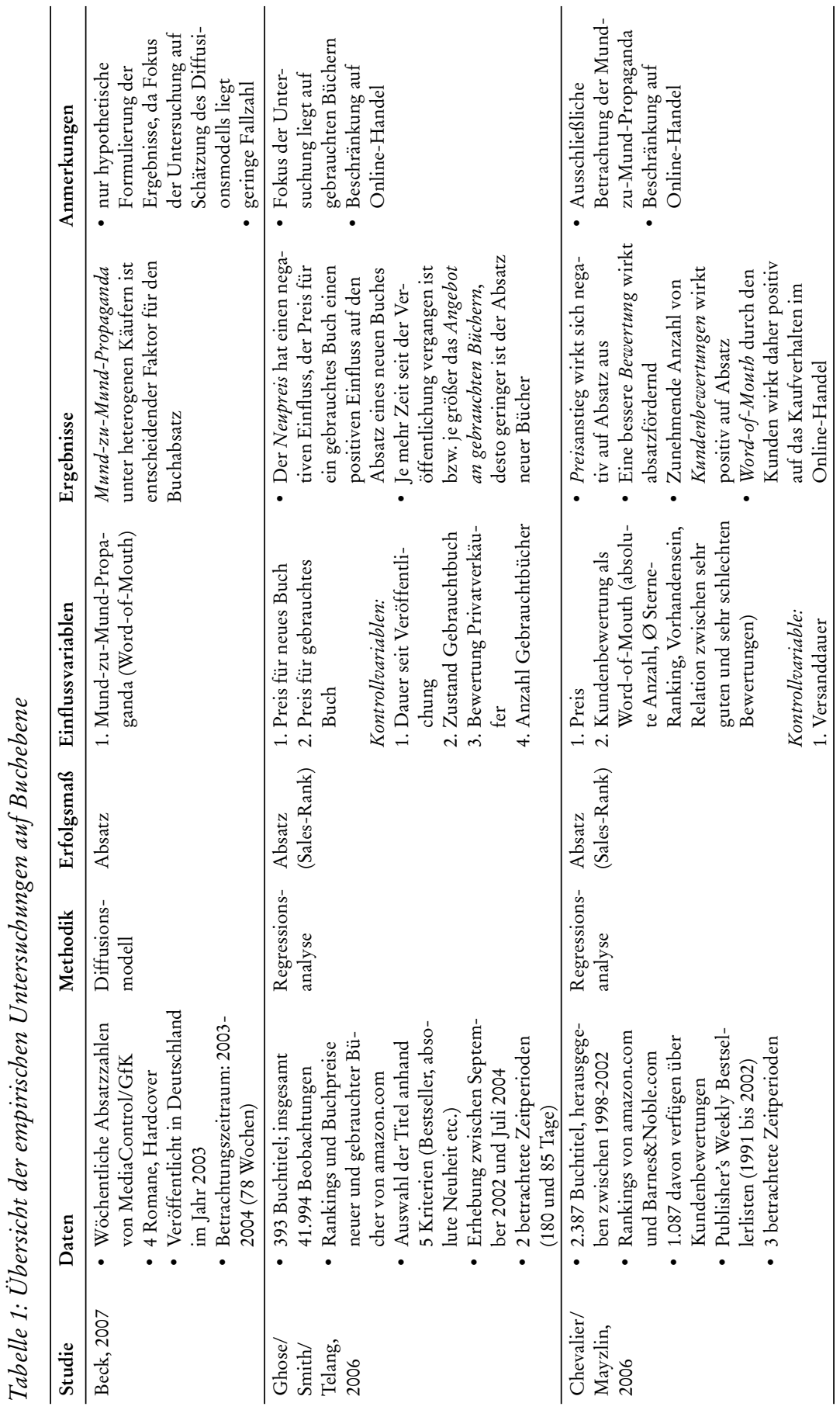




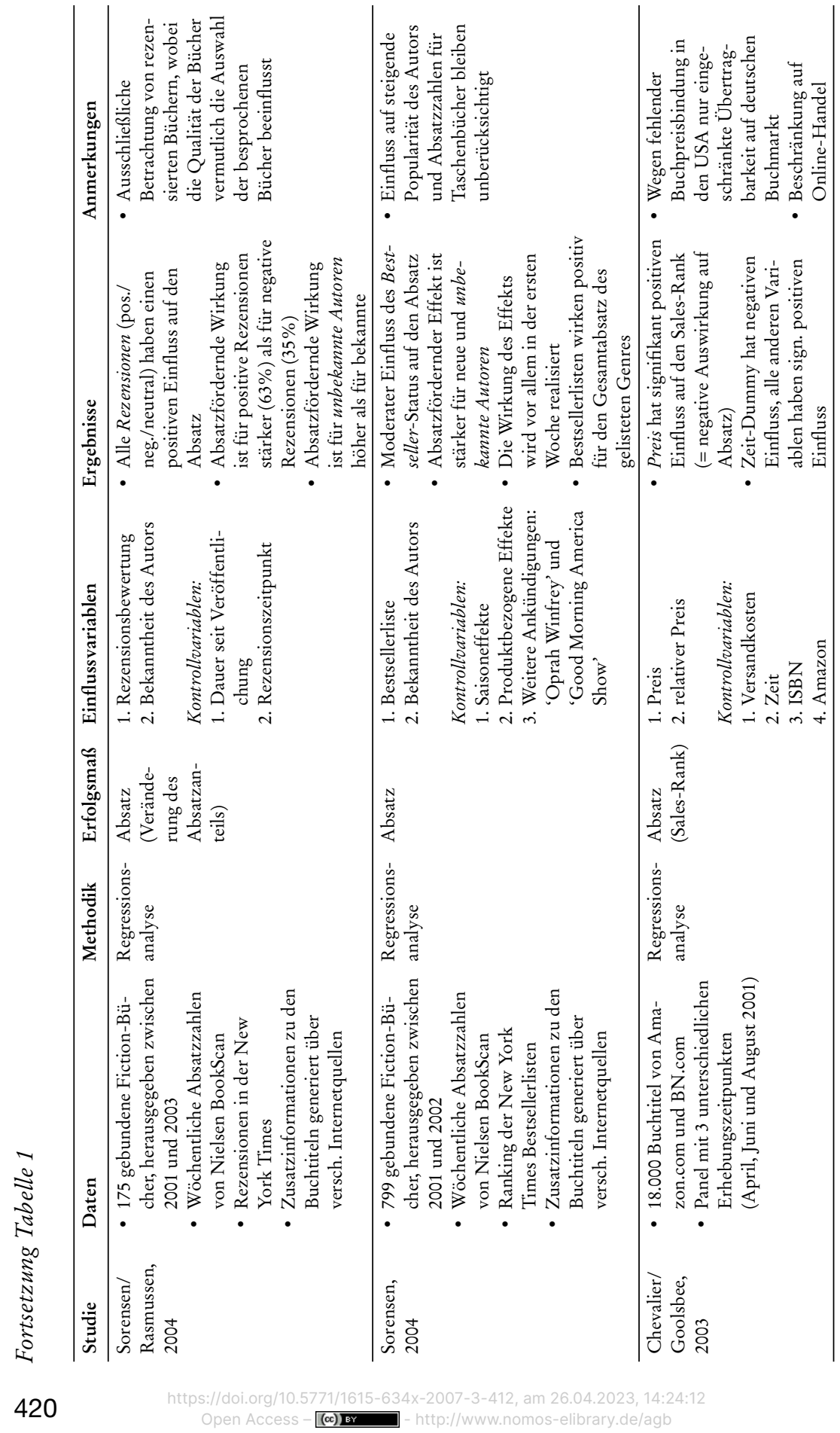




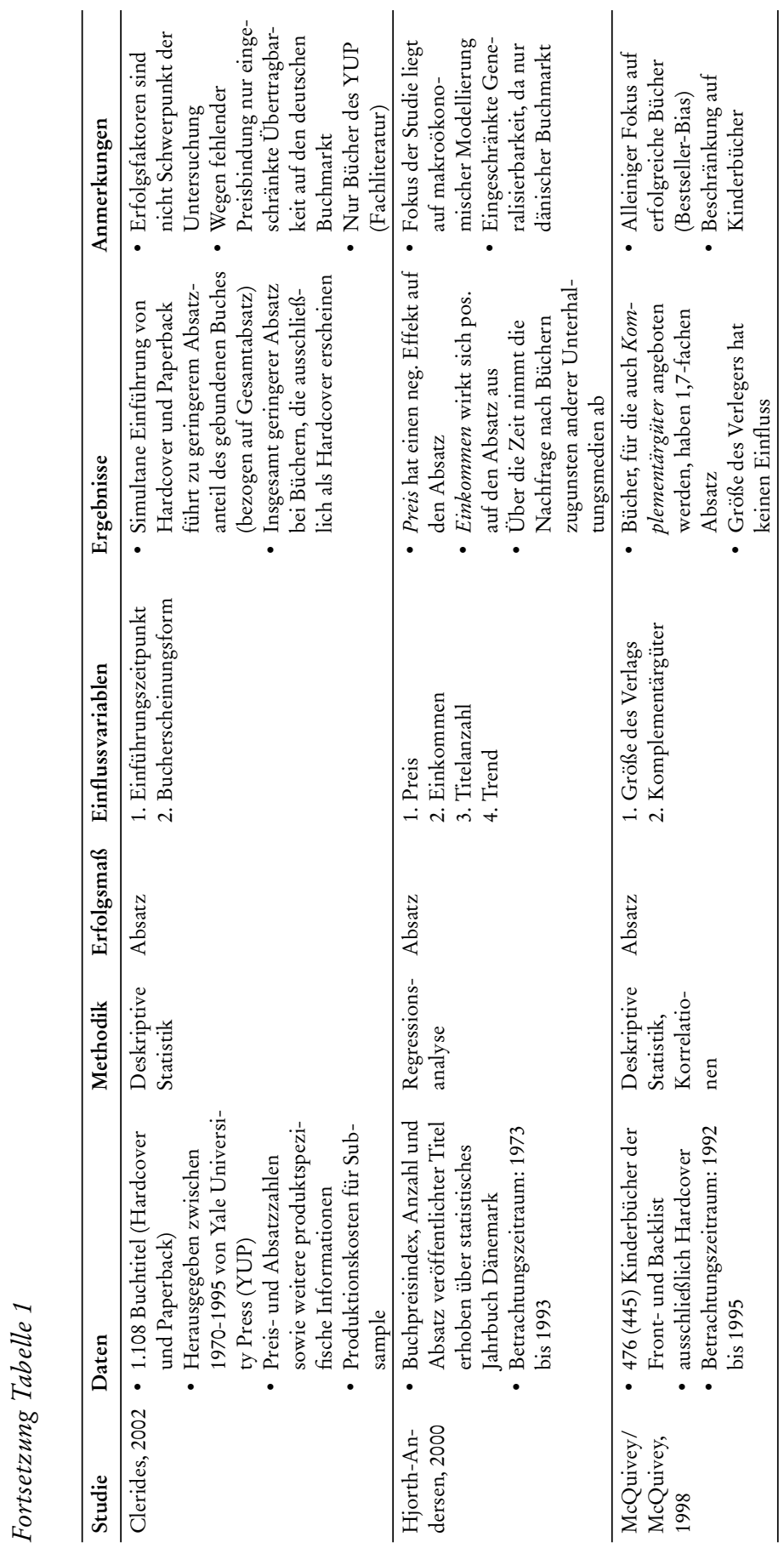




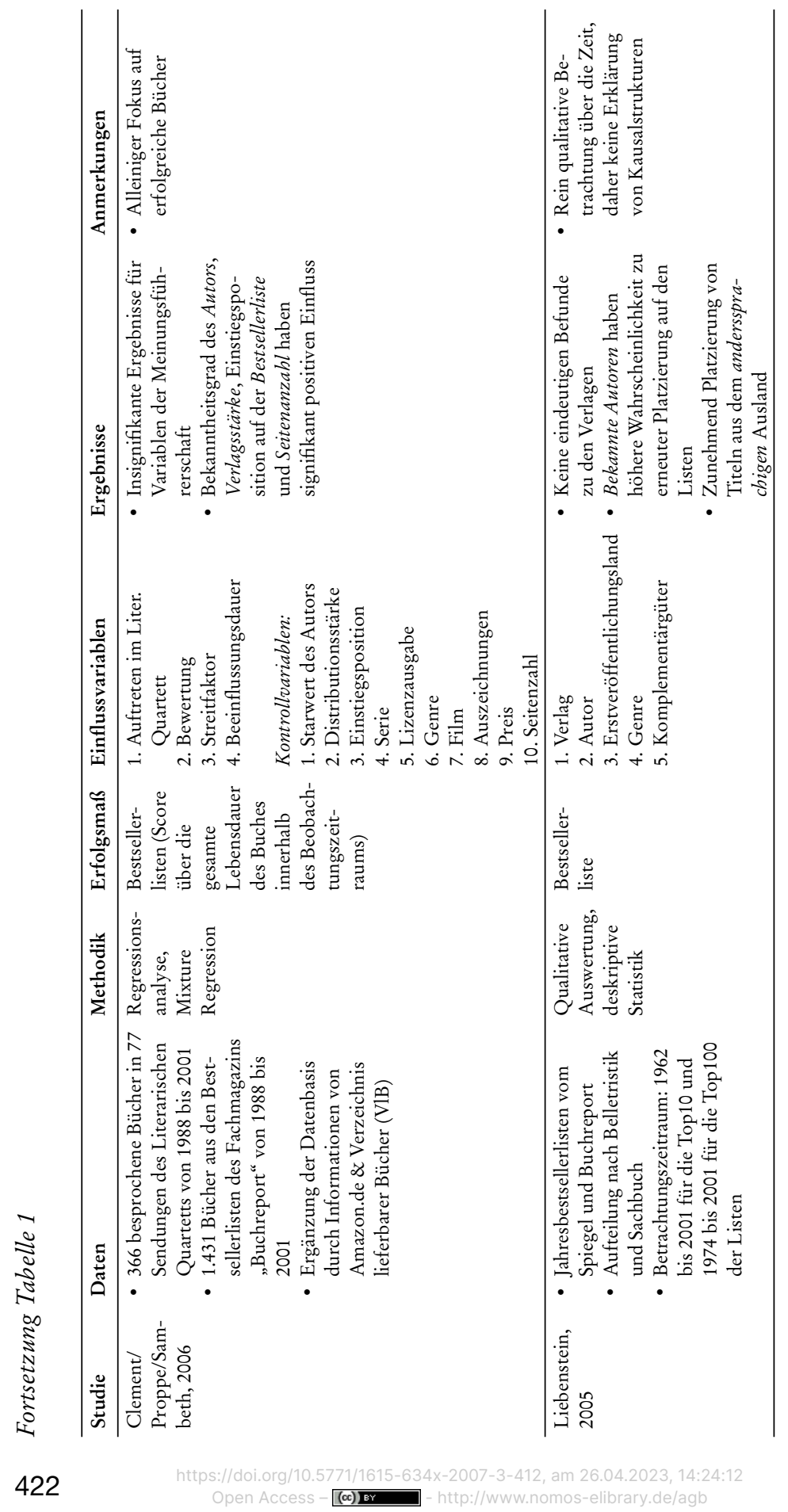




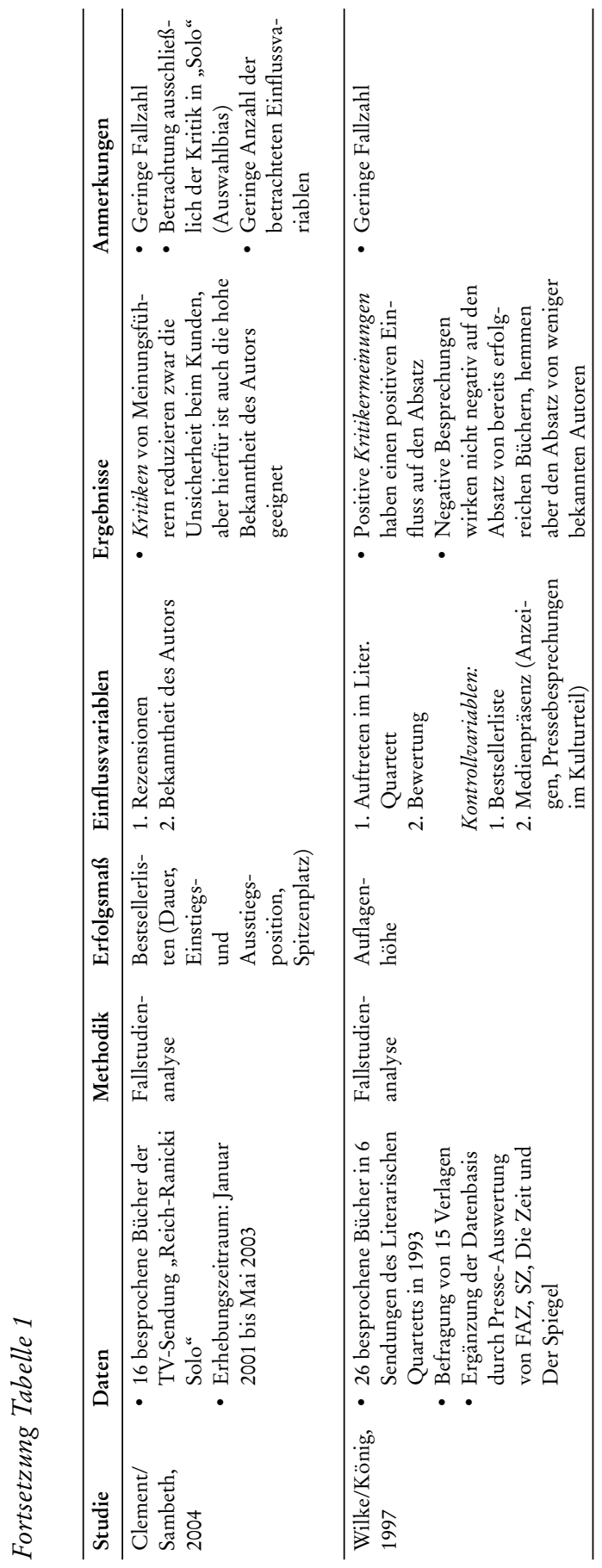




\section{Implikationen für das Management}

Die Ergebnisse lassen sich entlang der vier Elemente des Marketing-Mix gliedern: Produkt-, Preis-, Kommunikations- und Distributionspolitik. Es wird deutlich, dass der Produkt- und Kommunikationspolitik eine entscheidende Rolle in der Buchbranche zukommt. Die direkte Einflussnahme ist für den Verlag im Produktbereich am höchsten, da er - zusammen mit dem Autor - die Eigenschaften eines Buchs bestimmt. Mit Ausnahme von Werbung oder PR können viele Variablen der Kommunikationspolitik (z. B. Mund-zu-Mund-Propaganda) nicht unmittelbar beeinflusst werden. Der Verlag sollte hier steuernd wirken oder zumindest Impulse senden, da die Bedeutung der Kommunikationsmaßnahmen hoch ist. Die Buchpreisbindung schränkt die Möglichkeiten im Rahmen der Preispolitik für deutsche Verlage stark ein. Dennoch ist die Situation differenziert zu betrachten, da der Verkaufspreis für den Verlag - wenn auch nur hauptsächlich zum Erscheinungstermin und daher weniger flexibel - eine Komponente darstellt, die ausschließlich durch ihn determiniert werden kann.

\subsection{Einflussfaktoren der Produktpolitik}

Das Produkt - in diesem Falle das Buch als hedonisches Gut - ist ein Bündel an Eigenschaften (Brockhoff, 1993: 15ff.). Die Menge der Eigenschaften wird durch die Anbieter - hier die Verlage - gebündelt. Dabei stellen Thema und Inhalt des Buches (d. h. Text, Grafik, Illustration) den Produktkern dar und bilden somit den originären Nutzen für den Konsumenten (Abbildung 3). ${ }^{4}$ Aufgrund der Unsicherheit bei hedonischen Gütern sind für den Kunden weitere produktbezogene Signale relevant. Dazu bieten sich

\section{Abbildung 3: Produktkomponenten des Buches}

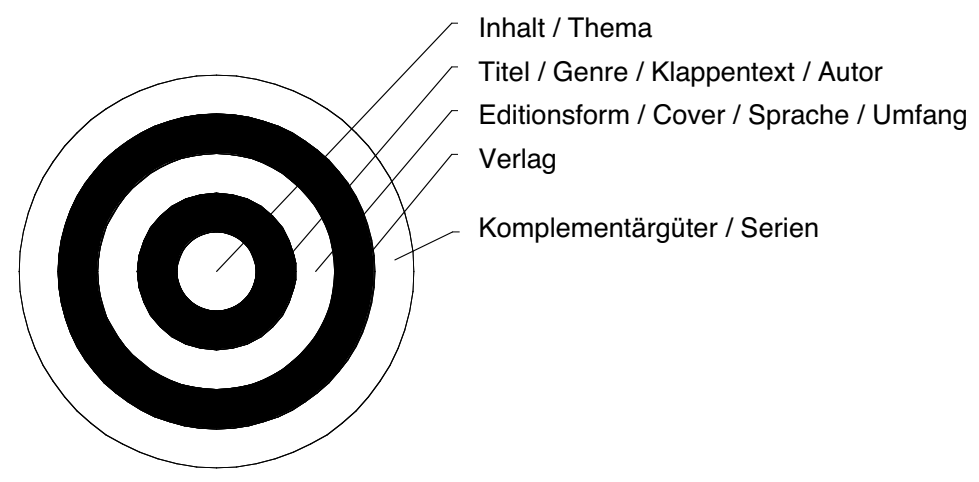

4 Es kann allerdings auch argumentiert werden, dass die Sprache, die in Abbildung 3 nicht zum Kern gezählt wird, dem Produktkern zuzuweisen ist, da der Verlag normalerweise das Recht für die Herausgabe von Büchern in einer spezifischen Sprache (bzw. bei Englisch noch weiter differenziert in ein Verbreitungsgebiet) erwirbt. Wir nehmen daher die Produktsicht vor der Internationalisierung des Buches an. 


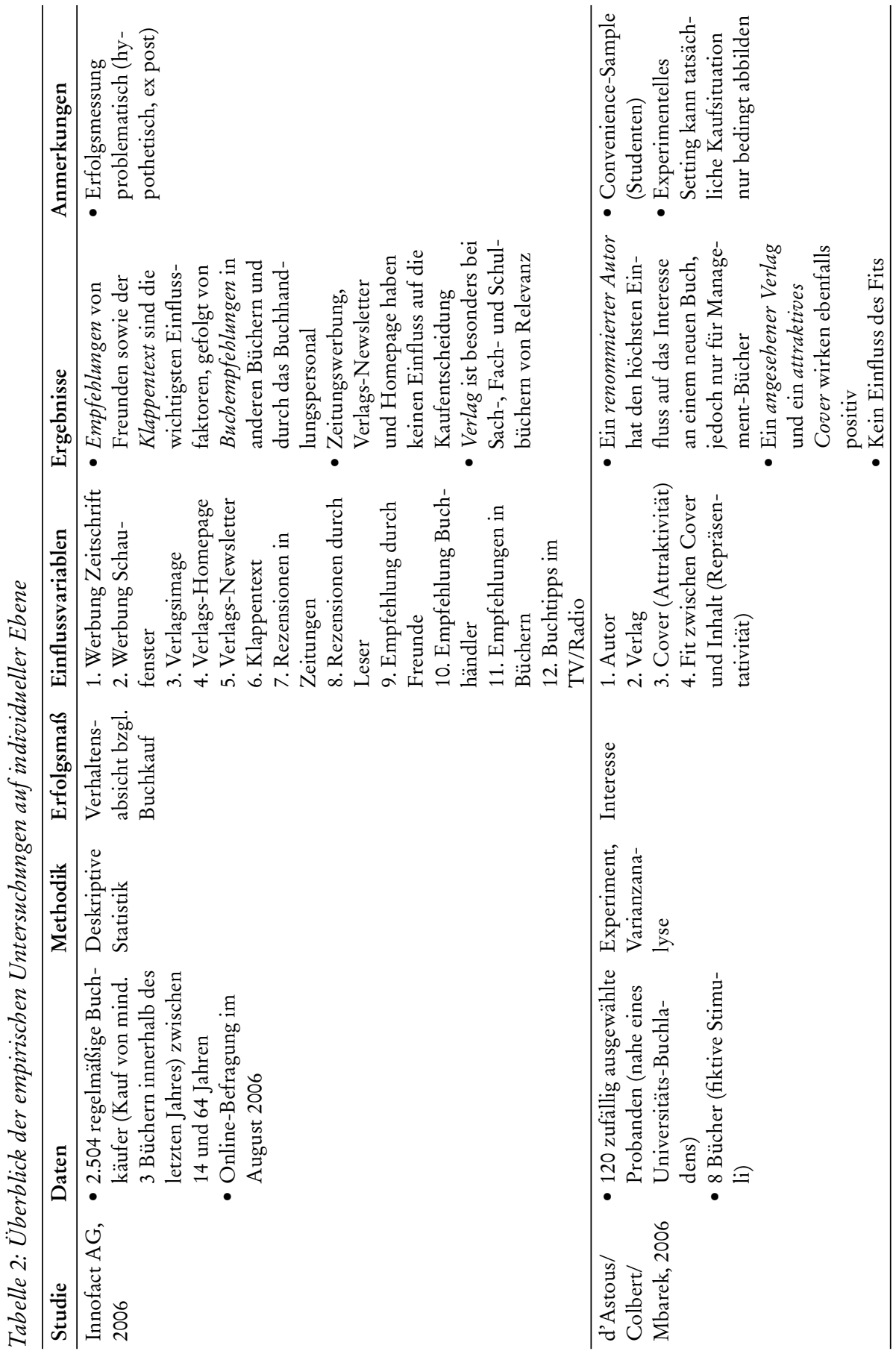




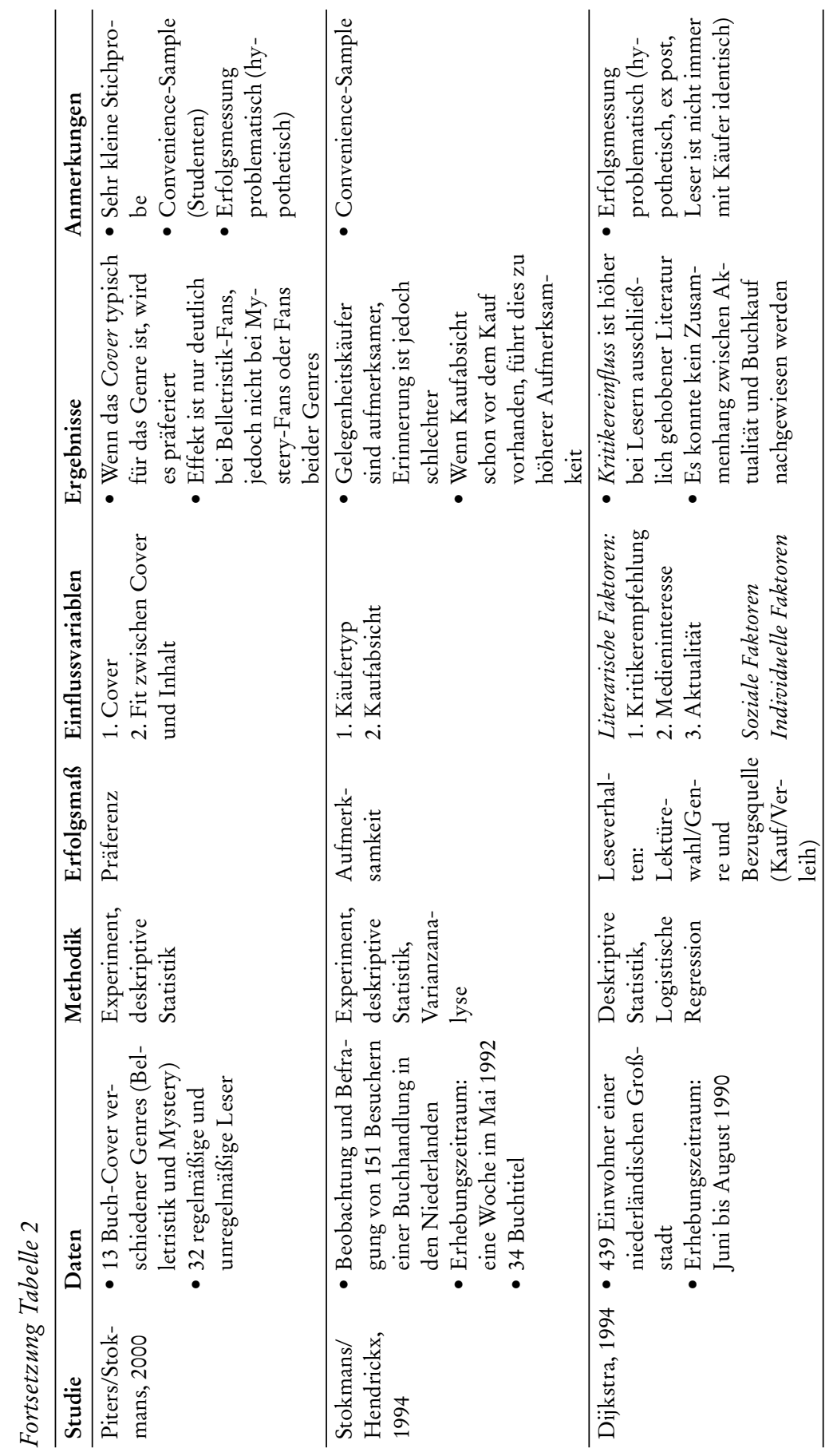



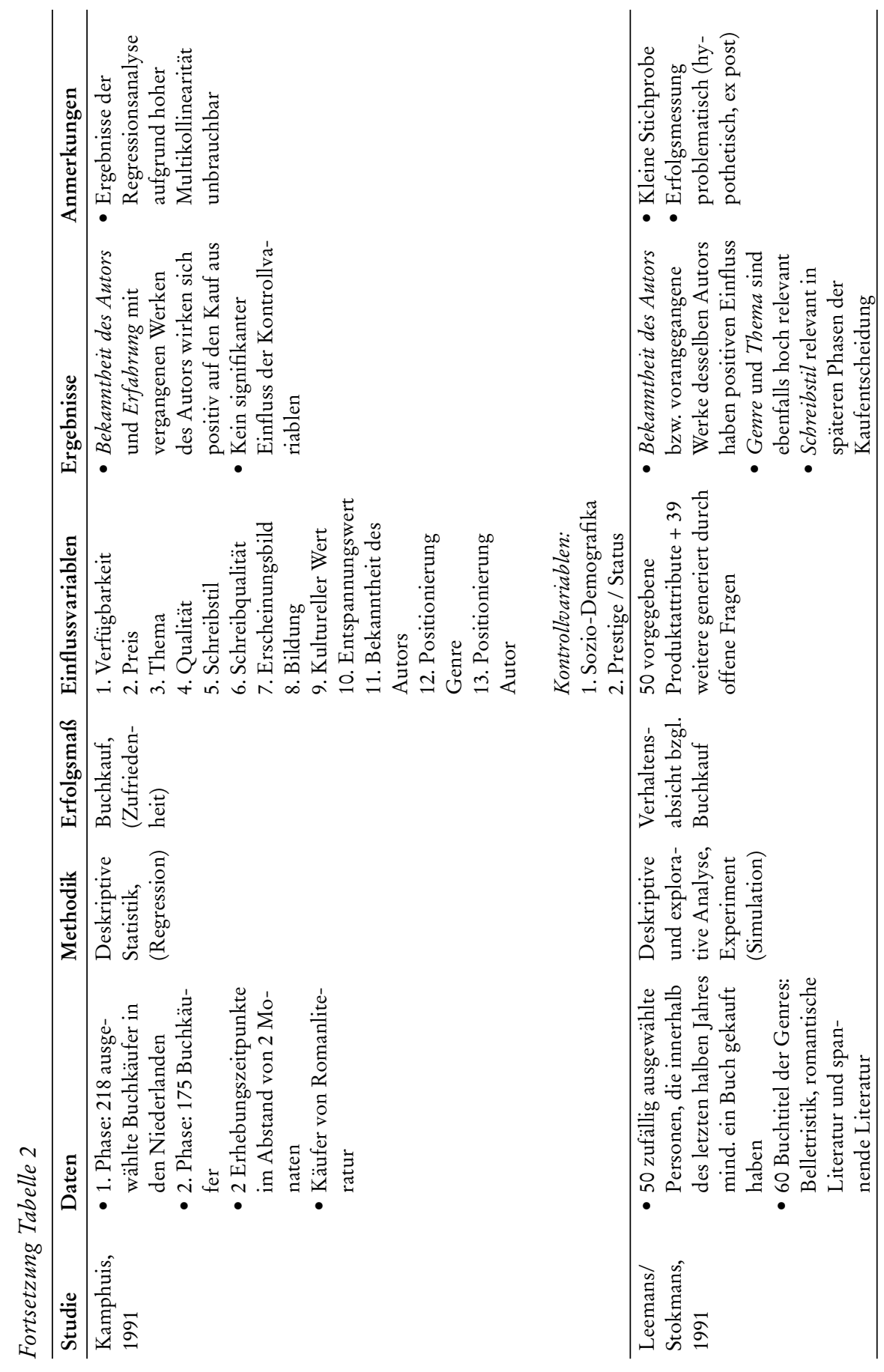


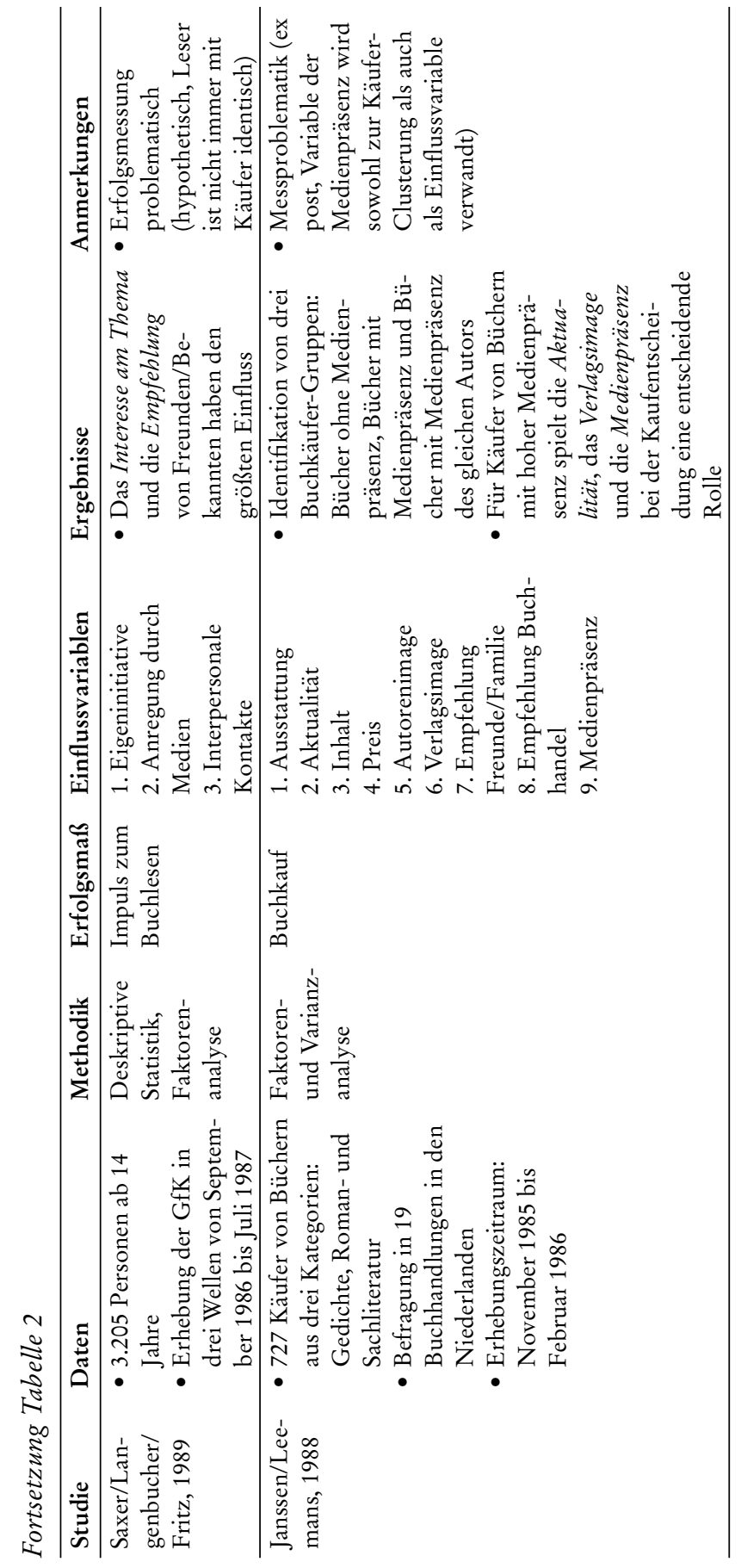


auf einer zweiten Ebene Aspekte an, die das Buch und seinen Inhalt näher beschreiben. Hier spielen der Titel, das Genre und der Klappentext sowie der Autor eine große Rolle. Zum hedonischen Nutzen gehören ebenso Merkmale, die das äußere Erscheinungsbild des Produktes betreffen, wie das Cover oder die Editionsform (Hardcover versus Paperback). ${ }^{5}$ Auf einer letzten Ebene wird das Buch durch den Verlag, bei dem es erschienen ist, determiniert. Darüber hinaus gibt es Merkmale, die nur indirekt mit dem entsprechenden Kernprodukt verbunden sind. Für den Buchbereich können Komplementärgüter oder Serien als Beispiele angeführt werden. Diese Untergliederung der Produktkomponenten soll in erster Linie jedoch zur Strukturierung der Darstellung der Erfolgsfaktoren dienen.

Die Aktualität des Inhaltes, d. h. die Auseinandersetzung des Verfassers mit Themen, welche die Gesellschaft zum Erscheinungszeitpunkt bewegen, kann eine positive Wirkung auf den Erfolg eines Buches haben (Noelle-Neumann/Schulz, 1987: 2157). Allerdings steht demgegenüber das Problem des schnellen Veraltens. Bezüglich der Aktualität gibt es nur sehr wenige Erkenntnisse aus den empirischen Studien. Ein Grund kann in der problematischen Operationalisierung dieses Faktors auf Buchebene gesehen werden, so dass die Aktualität häufig nicht in die Regressionsanalysen einbezogen wird. Die Ergebnisse der Studien auf individueller Ebene deuten jedoch darauf hin, dass es sich um einen wichtigen Aspekt beim Buchkauf handelt.

Das Thema eines Buches gibt ebenfalls einen Anstoß zum Buchkauf bzw. -lesen (Weinhold, 1956: 274). Das starke persönliche Themeninteresse kann auch als primäres Motiv zum Erwerb eines Buches gelten (Stiftung Lesen, 1993: 56). Dieser Einflussfaktor wurde bisher vor allem aus Sicht der Leseintensität und -häufigkeit betrachtet. Es liegen jedoch keine aktuellen betriebswirtschaftlichen Studien vor, die sich mit dem Merkmal auseinandersetzen - vor allem nicht auf Buchebene, da dies eine Erhebung der Buchthemen für die gesamte Stichprobe erfordern würde. Allerdings ist dieser Faktor für das Verlagsgeschäft von hoher Relevanz und sollte verstärkt in die Forschung einbezogen werden.

Die Bekanntheit eines Autors dient den Nachfragern als Signal für die Qualität und die Eigenschaften des Erfahrungsgutes „Buch“ (Clement/Sambeth, 2004: 8; Tietzel, 1995: 41). Die Markenbildung, die von Verlagen mit und durch Autoren betrieben wird, bindet Autoren langfristig an sie und schafft eine identifizierbare Einheit im Markt. Viele Autoren (z. B. Frank Schätzing) sind längst durch Erfolge der Vergangenheit sowie Marketingmaßnahmen zu „Marken“ geworden (Basuroy/Chatterjee/Ravid, 2003: 104; Ludes, 1989: 28).

Da hedonische Produkte zudem eine Symbolfunktion innehaben, ist das Konsumrisiko besonders hoch, das falsche Buch zu wählen. So ist es für viele Buchkäufer ein Element der sozialen Positionierung, welchen „kulturellen“ Buchstil sie symbolisieren und welche Autoren sie bewundern (Basuroy/Chatterjee/Ravid, 2003). Daher ist es wichtig, die „richtigen“ Bücher bzw. Autoren innerhalb des eigenen sozialen Netzes (z. B. Grass in "gehobenen Kreisen“) zu kennen. Diese hohe soziale Komponente der Literatur begründet auch die starke Orientierung an den Bestsellerlisten (Bradlow/Fader, 2001). Aus der hedonischen Komponente des Buches lässt sich ebenfalls das Vorliegen von Netzeffekten begründen; denn je mehr Menschen den selben Autor lesen, desto geringer

5 Alternativ können auch die Ebenen 2 (Titel, Genre etc.) und 3 (Editionsform, Cover etc.) zusammengefasst werden. Allerdings basiert unsere Argumentation auf der Trennung zwischen inhaltsbezogenen versus trägerbezogenen Eigenschaften, die jedoch nicht immer trennscharf sind. 
ist das soziale Risiko für den Einzelnen, ein möglicherweise falsches Signal nach außen zu senden.

Daher wurde seitens der Verleger der Einfluss der Reputation eines Autors auf die Planbarkeit und Höhe der Absatzzahlen schon immer als hoch eingeschätzt (Schweitzer, 1997: 26; Tietzel, 1992: 322). Diese Hypothese kann empirisch belegt werden: Clement/ Proppe/Sambeth (2006) sowie Liebenstein (2005) zeigen den positiven Einfluss des Bekanntheitsgrades eines Autors auf die Platzierung in der Bestsellerliste. $\mathrm{Zu}$ ähnlichen Ergebnissen kommen auch d'Astous/Colbert/Mbarek (2006) sowie Kamphuis (1991), die jeweils den Einfluss des Renommees auf Interesse bzw. Kaufverhaltensabsicht untersucht haben. Ebenso wird die absatzfördernde Wirkung von Rezensionen besonders für unbekannte Autoren bestätigt (Sorensen, 2004: 17; Sorensen/Rasmussen, 2004: 11).

Eng mit der Bekanntheit verknüpft ist die Person des Autors. Die (nationale, religiöse oder ethnische) Herkunft des Autors kann ebenfalls für bestimmte Kundensegmente von Bedeutung sein. Auch finden sich Biografien immer wieder in den Bestsellerlisten. Der Verkaufserfolg dieser Bücher ist umso sicherer, je radikaler die Biografie im Widerspruch zum bekannten Bild steht (Czepek, 2001: 90). Während die Bekanntheit des Autors mehrfach empirisch untersucht worden ist, gibt es bisher lediglich theoretische Überlegungen zur Wirkung von Person und Herkunft des Autors sowie dem Einfluss von (Auto-)Biografien.

Insgesamt lässt sich festhalten, dass vor allem die theoretische Forschung dem StarWert von Autoren ein hohes Gewicht zuweist, welches teilweise in den neueren empirischen Studien auch nachgewiesen werden kann (Clement/Proppe/Sambeth, 2006). Allerdings gilt es zu beachten, dass Stars in der Lage sind, ihren Markenwert über Vorauszahlungen bzw. Garantiehonorare zu monetarisieren, so dass zwar der Absatz bzw. Umsatz höher ist, aber zugleich auch die Kosten steigen. Da bislang empirische Ergebnisse bezüglich der Gewinnwirkung fehlen, lassen sich im Gegensatz zur Filmindustrie noch keine konkreten Aussagen ableiten (Clement, 2004).

Das Sachgebiet ist insbesondere bezüglich des Kaufanlasses von Relevanz. So zeigen Leserstudien, dass Bildbände, Dokumentationen etc. eher als Geschenk, Fachbücher hingegen vor dem Hintergrund der Weiterbildung gekauft werden. Diese unterschiedlichen Nutzungsgewohnheiten werden vom Verlagsmanagement im Rahmen der Produktpolitik zunehmend häufiger adressiert, z. B. durch Steigerung der „Wertigkeit“ in den jeweiligen Genres in Form von aufwändigen Geschenkbänden. Darüber hinaus ist eine eindeutige Genre-Zuordnung nicht immer möglich. In einer experimentellen Studie konnte gezeigt werden, dass die Probanden - trotz Word-of-Mouth und identischen Nutzungsgewohnheiten - gelesene Bücher unterschiedlich klassifizieren bzw. verschiedenen Subkategorien zuordnen (Stokmans, 2002). Dies muss bei der Planung von Marketingaktivitäten berücksichtigt werden.

Wenige Studien betrachten die Relevanz des Buchtitels. Dabei ist festzustellen, dass häufig nur ein geringer oder gar kein Einfluss auf den Buchabsatz beobachtet wird (Bonfadelli/Fritz, 1993: 159; Link/Hopf, 1946: 108). Es gibt keine neueren Untersuchungen zu diesem Themengebiet, so dass die bisherigen Ergebnisse kaum generalisierbar sind - allerdings unterstützt ein "griffiger" Titel den Leser bei der Einordnung des Buches in Genre-Kategorien.

Eine entscheidende Rolle für die Kaufentscheidung kann auch die Bucherscheinungsform spielen. Nach Meinung der Buchhändler nimmt das Taschenbuch eine nahezu gleichwertige Stellung neben der traditionellen Hardcoverausgabe ein (Martin/Scheer, 1995: 36ff.) - besonders Vielleser präferieren das Taschenbuch (Börsenverein, 2005b: 11). Speziell für das Genre Belletristik entfällt mehr als die Hälfte der Umsätze auf die Ta- 
schenbuchausgabe (Börsenverein, 2007: 8). Werden die Bucherscheinungsformen Hardcover und Paperback gleichzeitig herausgebracht, dann beträgt der Anteil der verkauften gebundenen Ausgabe im US-amerikanischen Markt nur 12\% am gesamten Absatz. Bei der späteren Herausgabe der Taschenbuchausgabe steigt der Anteil der Hardcoverausgabe auf ca. 38\%. Der Absatz der Bücher, die ausschließlich in gebundener Ausgabe erschienen sind, ist geringer als der Absatz bei Herausgabe von Hardcover und Paperback (Clerides, 2002: 1390ff.). Dies verdeutlicht die Relevanz der Preisdifferenzierung, die hier stark mit der Produktdifferenzierung gekoppelt ist. Die gängige Praxis vieler Verlage, zunächst eine Hardcover- und später eine Paperbackausgabe auf den Markt zu bringen, stellt demnach eine sinnvolle Strategie dar.

Weiterhin kommt der Gestaltung des Covers eine große Bedeutung zu. Ein ansprechendes Layout wird von den Verbrauchern erwartet (Köcher, 1988: 298f.). In den meisten Fällen lässt sich ein Buch anhand des Covers der entsprechenden Buchgattung zuordnen, was die Entscheidung für den Konsumenten erleichtern und so den Erfolg steigern kann (Piters/Stokmans, 2000: 159). Inwieweit die Käufer ähnlich anmutende Cover als eine einheitliche Dachmarke erkennen (z. B. ähneln sich die Cover bei bestimmten Serien), bleibt jedoch bisher offen.

Auf dem Buchmarkt ist die Rolle der Sprache nicht zu unterschätzen. Immer mehr US-Verlage entdecken beispielsweise den spanischsprachigen Buchmarkt für sich (Sieg, 2003: 36f.). Ebenso lässt sich für den englischsprachigen Markt in Deutschland ein hohes Potenzial vermuten. Der Einfluss der Sprache ist bisher jedoch empirisch noch nicht untersucht worden. Gleiches gilt für den Umfang eines Buches. Lediglich Clement/ Proppe/Sambeth (2006) haben bisher die Beziehung zwischen Seitenzahl und Buchabsatz näher beleuchtet.

Einen positiven Einfluss auf den Erfolg eines Buches können Komplementärgüter haben. Das Buch eines Autors kann in eine Literaturverfilmung „umgesetzt" werden, die dann den Verkauf des Buches und die Popularität des Autors fördert. Der Bestsellerautor John Grisham wurde literarisch erst bekannt, nachdem er die Filmrechte an seinem Roman „Die Firma“ verkauft hatte (Hoffmann, 1994: 108ff.). Durch Nebenprodukte wie Spielfilme können Konsumenten positiv beeinflusst werden, das Buch zu kaufen oder zu lesen (Köcher, 1993: 297). Dieser Zusammenhang gilt auch für den Markt der Kinderbücher (McQuivey/McQuivey, 1998: 41ff.) - populärstes Beispiel ist hier die „Harry-Potter"-Buchserie von Joanne Rowling mit ihren Verfilmungen und unzähligen Merchandising-Artikeln. Ein sehr erfolgreiches Beispiel im deutschen Markt ist der Coppenrath Verlag (Maskos, 2006).

Der amerikanische Kinderbuchmarkt wird nicht nur von Harry Potter und den Newbery- und Carnegie-Preisträgern besetzt, sondern auch immer wieder von Serien. Auf dem US-Buchmarkt wurden 13,2 Mio. „Chicken-Soup“-Ratgeber für Teenager, 3,6 Mio. „Left-Behind“-Thriller mit religiösem Hintergrund und 2,5 Mio. „MagicTree-House“-Bücher für die jüngsten Leser verkauft (Sieg, 2001: 127). Die Folgebücher profitieren von den bereits aufgebauten Autorennamen, dem Verlagsimage und der Bekanntheit des Themas (Brown, 2001: 472ff.). Clement/Proppe/Sambeth (2006) zeigen, dass Serien nur in einem mäßig erfolgreichen Segment einen signifikanten Einfluss auf die Bestsellerlistenplatzierung haben. 


\subsection{Einflussfaktoren der Kommunikationspolitik}

Wie der Vergleich der hohen Anzahl von Neuerscheinungen mit dem begrenzten Angebot in den Buchvertriebskanälen deutlich macht, reicht die bloße Herausgabe von Büchern nicht aus, um diese an die Nachfrager zu bringen. Vielmehr ist eine gezielte Kommunikationspolitik notwendig, die die publizierten Bücher aus der Masse hervorhebt. Die Ausgaben für kommunikationspolitische Maßnahmen liegen in der Buchbranche zwischen acht und fünfzehn Prozent des Umsatzes (Vogel, 2004: 317; Wirtz, 2000: 202). Dabei werden drei verschiedene Zielgruppen adressiert: Buchkäufer und -leser, Literaturkritiker bzw. die Presse sowie der Handel.

Grundsätzlich wird für viele Verlagsprodukte Werbung geschaltet, um auf ihre Existenz aufmerksam zu machen (Stockem, 1988: 24). Die Verlage rücken dabei meist Autor und Buchtitel in den Mittelpunkt (o. V., 2003: 37). Werbung in Radio und Fernsehen wird für Bücher nur in Einzelfällen genutzt - die klassischen Medien sind hier direkt die Verkaufsstätte (Point-of-Sale), Anzeigen in Zeitungen und Zeitschriften und Kataloge von traditionellen und Internet-Versendern. Für Buchhändler spielen hingegen Informationsquellen wie Verlagsverzeichnisse, das Verkaufsgespräch mit einem Vertreter sowie weitere direkte Kommunikationsmaßnahmen der Verlage eine große Rolle. Eine spezielle Werbeform stellt die Bestsellerwerbung dar, bei der das Buch gezielt über die Nennung von Autor und Bestsellerlistenrang positioniert wird (Lauterbach, 1979: 95ff.; Sorensen, 2004). Die zentrale Fragestellung für einen Verlag betrifft hier die optimale Allokation des Werbebudgets. Bei der Vielzahl der Bücher im Verlagsprogramm muss eine Auswahl stattfinden, so dass bestimmte Bücher gezielt beworben werden können. Darüber hinaus ist auch das gewählte Werbemedium relevant, da die empirischen Untersuchungen hier durchaus konträre Ergebnisse zeigen. So spielt insbesondere die Werbung in Zeitungen oder über Newsletter und die Verlagshomepage im Entscheidungsprozess des Konsumenten nur eine untergeordnete Rolle (InnofactAG, 2006: 25).

Ein weiteres Mittel zur Verkaufsförderung sind Leseexemplare. Vom Versand kostenfreier Leseexemplare erhoffen sich die Verlage; ihre Autoren ins Gespräch zu bringen und die Anzahl der Vorbestellungen zu erhöhen (Ebbinghaus, 2003: 13f.). Dieser Bereich wurde bisher in der empirischen Forschung noch nicht behandelt, so dass unklar ist, wie groß der Einfluss dieses Instruments auf den Buchabsatz oder allgemein auf den Bucherfolg tatsächlich ist. Darüber hinaus adressieren Leseexemplare hauptsächlich den Handel und nicht den Endkunden.

Die Bestsellerliste, die in einigen Studien als Erfolgsmaß verwendet wird, sorgt als Einflussvariable ebenfalls für Verkaufserfolg und vor allem höhere Gewinne (Ziermann, 2000: 110). Buchkäufer müssen sich in einem unübersichtlichen Buchangebot zurechtzufinden und Bestellerlisten erleichtern die Orientierung, da sie als Qualitätsindikator dienen (Saxer/Langenbucher/Fritz, 1989: 127). Die Bestsellerliste der New York Times hat einen direkten Einfluss auf den Absatz der Bücher und bringt eine Absatzsteigerung von ca. 14\%, für unbekannte Autoren sogar bis zu 57\% (Sorensen, 2004: 17f.). Hier sind jedoch auch Endogenitätseffekte zu erwarten, da bei bekannten Autoren die Wahrscheinlichkeit der Listen-Platzierung höher ist (Clement/Proppe/Sambeth, 2006).

Öffentlich verliehene Preise und Auszeichnungen stellen ebenfalls einen Qualitätsmaßstab dar und dienen zur Orientierung der Kunden (Leemans/Stokmans, 1991: 501). Die Preisverleihung kann zudem die Bekanntheit eines Autors steigern und somit über den Stareffekt auch indirekt wirken (Faulstich, 1983: 176f.; Wilke/König, 1997: 260). Clement/Proppe/Sambeth (2006) zeigen den Einfluss nationaler und internationaler Auszeichnungen auf (z. B. Nobelpreis, Pulitzerpreis für Literatur) - allerdings wird ein 
signifikanter Einfluss nur in einem von insgesamt drei Kundensegmenten offenbar, so dass die Relevanz dieses Faktors nicht überschätzt werden sollte. Des Weiteren unterliegt dieser Faktor nicht dem unmittelbaren Einfluss des Verlages.

Als weitere verkaufsfördernde Faktoren gelten Rezensionen. Die Glaubwürdigkeit der Rezensionen ist in der Reputation des Rezensenten begründet, der mit der Empfehlung keine kommerziellen Interessen verfolgt. Eine positive Kritik eines bekannten Kritikers führt bei bekannten Autoren wie Grass, Walser, Roth oder Updike nicht unbedingt zu höheren Verkäufen. Von Seiten der Kritiker kann aber eine gute Prognose über den (Miss-)Erfolg eines Buches gegeben werden (Clement/Sambeth, 2004: 109ff.). Dijkstra (1994) zeigt, dass auf individueller Ebene Kritiker besonders bei Lesern anspruchsvoller Literatur einen hohen Einfluss haben. Sorensen/Rasmussen (2004) untersuchen den Wert einer Rezension in der New York Times und die Höhe des Einflusses einer positiven bzw. negativen Kritik auf den Bucherfolg. Für relativ neue Autoren ist der Einfluss höher als für etablierte Schriftsteller, da eine der wichtigsten Funktionen von Besprechungen die Informationsfunktion ist (entweder über die Herausgabe eines Buches oder über seine Merkmale). Für Buch-Ankündigungen im „Oprah Winfrey Book Club" sowie in der "Good Morning America Show" wurde ein starker positiver Einfluss auf den Absatz nachgewiesen (Sorensen, 2004: 30). Es bleibt festzuhalten, dass die theoretische und empirische Forschung im Hinblick auf Rezensionen teilweise zu konträren Ergebnissen kommt (Clement/Proppe/Sambeth, 2006). Insbesondere bei Verlegern und Buchhändlern liegt eine Überbewertung dieses Einflusses vor (Wilke/ König, 1997: 279), denn aus der Perspektive der Buchkäufer scheint von Rezensionen eher ein Informationseffekt und weniger ein Überzeugungseffekt auszugehen (Martin/ Scheer, 1995: 36ff.).

Ein weiterer Einflussfaktor der Kommunikationspolitik ist die Mund-zu-MundPropaganda (Word-of-Mouth). Die Empfehlungen von Freunden und Bekannten sind für viele Buchkäufer das wichtigste Kriterium bei ihrer Entscheidung (Fritz, 1989; Harmgarth, 1999; Saxer/Langenbucher/Fritz, 1989; Schweitzer, 1997). Diese Erkenntnis gilt unabhängig von der Leseintensität (Bonfadelli/Fritz, 1993: 159). Der Einflussfaktor Word-of-Mouth ist jedoch bisher kaum in Bezug auf den Buchabsatz untersucht worden. Beck (2007) unterstellt zwar in seinem Diffusionsmodell eine positive Beziehung, nimmt jedoch keine empirische Überprüfung dieser Hypothese vor.

Im Rahmen der Mund-zu-Mund-Propaganda wurde in einer aktuellen Studie zum Online-Buchmarkt der Faktor Kundenbewertungen berücksichtigt (Chevalier/Mayzlin, 2006). Hier fallen Rezensions- und Word-of-Mouth-Effekte zusammen und es konnte ein positiver Einfluss auf den Absatz festgestellt werden. Dies gilt sowohl für positive Beurteilungen als auch für eine zunehmende Anzahl an Bewertungen für ein bestimmtes Buch.

\subsection{Einflussfaktoren der Distributionspolitik}

Der Vertriebskanal spielt beim Buchkauf eine große Rolle (Dijkstra, 1994: 57; Kamphuis, 1991: 481; Köcher, 1993: 297; Schmidtchen, 1974: 765). Die Präsenz eines Buches im stationären Handel ist kritisch für den Kaufprozess, dieser Einflussfaktor wurde jedoch in den neueren empirischen Studien kaum untersucht. Auch wenn sich einige aktuelle Arbeiten auf den Internethandel beziehen und dazu Absatzzahlen von Online-Buchhändlern analysieren (Chevalier/Mayzlin, 2006; Chevalier/Goolsbee, 2003; Ghose/Smith/Telang, 2006), bleibt die Betrachtung jedoch immer isoliert, so dass keine Aussagen bezüglich der Unterschiede zwischen den einzelnen Absatzkanälen gemacht 
werden können. Untersuchungen zum Kaufverhalten bei Büchern zeigen, dass es bestimmte Präferenzen bei der Wahl der Kauforte gibt (Chevalier/Goolsbee, 2003). Daher ist zu vermuten, dass nur durch eine möglichst breite Verfügbarkeit in vielen unterschiedlichen Kanälen die Erreichbarkeit aller potenziellen Kunden gewährleistet ist und dies somit den Absatz und damit den Erfolg eines Buches fördert. Erste explorative Studien versuchen, unterschiedliche Präferenzen beispielsweise vor dem Hintergrund der Sinusmilieus zu erklären (Börsenverein, 2006c: 14ff.).

Mit zunehmender Relevanz des Internet-Handels ist auch die Versanddauer von Interesse. Diese Variable wird erstmals von Chevalier/Goolsbee (2003) berücksichtigt, die hierzu jedoch keine detaillierten Ergebnisse ausweisen. Zudem haben die Versandkosten, die im Online-Handel auftreten können, einen hohen Einfluss, denn die Preissensitivität ist hierbei sogar höher als bei den Produktpreisen (Smith/Brynjolfsson, 2001). Diese Faktoren können jedoch von Verlagsseite nicht beeinflusst werden und sind daher lediglich für den Handel relevant.

Die Verlagsgröße kann ebenfalls den Verkaufserfolg von Büchern beeinflussen, da größere Verlage eine stärkere Verhandlungsposition innehaben (Clement/Proppe/Sambeth, 2006: 10; Reibstein/Farris, 1995: G190). Im Kontrast dazu konnte Liebenstein (2005) keine Konzentration auf bestimmte Verlage bei ihrer Untersuchung der Bestsellerlisten feststellen. Hierbei handelt es sich jedoch um eine rein qualitative Auswertung. Auch McQuivey/McQuivey (1998) liefern konträre Ergebnisse zum erwarteten positiven Einfluss der Verlagsgröße auf den Bucherfolg. Die Autoren können die Befunde zumindest für den Spezialmarkt der Kinderbücher nicht bestätigen. Interessant wäre in diesem Zusammenhang eine Untersuchung, die das „Harry-Potter“-Phänomen in den letzten Jahren berücksichtigt, da hierdurch ein starker Impuls auf den Markt der Kinderund Jugendbuchliteratur ausging.

Äußerst kontrovers wird die Rolle des Verlagsimages als Träger der Markeneigenschaft in der Literatur behandelt. Während die Verlagsgröße primär den Handel tangiert, ist das Image eines Verlags auch für den Kunden relevant, so dass dieser Einflussfaktor ebenso als Instrument der Kommunikationspolitik klassifiziert werden könnte. Sowohl für Belletristik- als auch für Sach- und Fachbuchverlage stellt der Aufbau eines Verlagsimages bei den Käufern und Lesern eine der größten künftigen Herausforderungen dar. Durch die bisherige Werbung bzw. Presseaktivitäten werden jedoch eher die Bücher, Autoren und Themen und nicht die Verlage in das Bewusstsein der Konsumenten gebracht. Ein Verlagsimage ist bislang fast ausschließlich gegenüber dem Buchhandel aufgebaut worden (Schenkel, 1995: 23). Insbesondere für Fachbücher oder Bildbände ist jedoch zu erwarten, dass der Verlag bzw. dessen Reputation einen relevanten Faktor für die Kaufentscheidung darstellt. Eine aktuelle Studie (Innofact AG, 2006: 30f.) kann dies für die Warengruppen Sach-, Fach- und Schulbücher bestätigen, und auch die Ergebnisse weiterer Studien zielen in die gleiche Richtung (d'Astous/Colbert/Mbarek, 2006: 142). Janssen/Leemans (1988) zeigen darüber hinaus, dass die Reputation des Verlags besonders ausschlaggebend ist, wenn Kunden ihre Kaufentscheidung aufgrund der vorangegangenen Medienpräsenz des Buchtitels treffen. Unter Berücksichtigung der unterschiedlichen Relevanz für die verschiedenen Genres ist es für Verlage daher wichtig, Möglichkeiten der direkten Kundenansprache zu finden und sich entsprechend zu positionieren. Die Veröffentlichung von Sammeleditionen (so genannte „Billig-Bücher") ist ein Exempel, wie Zeitungshäuser in letzter Zeit versuchen, ihre Marktposition und ihr Image zu nutzen, um zusätzliche Erlösquellen zu generieren und somit ihre Position zu stärken. Dies kann als Beispiel dafür dienen, dass das Verlagsimage ein monetarisierbarer Erfolgstreiber sein kann. 
Der Zeitpunkt der Herausgabe eines Buches kann eine wichtige Rolle für den Verkaufserfolg spielen. Die saisonale Entwicklung der Buchumsätze lässt sich seit einigen Jahren beobachten (Börsenverein, 2006a: 29f.). Es gibt in der Literatur bisher keine empirischen Studien, die sich explizit mit diesem Einflussfaktor beschäftigt haben. Zu überprüfen wäre beispielsweise die Hypothese, ob ein zielgerichtet zum Weihnachtsgeschäft veröffentlichtes Buch höhere Erfolgschancen hat. Sowohl Chevalier/Goolsbee (2003) als auch Ghose/Smith/Telang (2006) beobachten einen sinkenden Absatz über die Zeit, während dieser Faktor bei Dijkstra (1994) keinen Einfluss auf das Leseverhalten hat. Hieran lässt sich gut verdeutlichen, dass die Verwendung unterschiedlicher Erfolgsmaße Einfluss auf die Ergebnisse und damit auch auf die Vergleichbarkeit hat. Möglicherweise sind hier auch die Ergebnisse aus dem Filmbereich übertragbar, die nahelegen, dass zwar in den Hochsaisonphasen eine höhere Nachfrage herrscht (z. B. Weihnachten), aber ebenfalls mehr Bücher veröffentlicht werden, so dass damit auch der Wettbewerb stärker ist (Radas/Shugan, 1998).

Ebenso ist das Timing zwischen Hardcover- und Paperback-Version kaum untersucht worden. Der Gesamtabsatz ist grundsätzlich höher, wenn beide Editionsformen publiziert werden (Clerides, 2002). Die zeitliche Abstimmung der beiden Editionsformen stellt eine zentrale Forschungsfrage dar, denn im Filmbereich wurde gezeigt, dass die Zeitspanne zwischen Kino- und DVD-Verwertung zu lang ist und so Spill-over-Effekte unterschätzt und Kannibalisierungseffekte überschätzt werden (Lehmann/Weinberg, 2000).

Ein weiterer Einflussfaktor, der empirisch bisher vernachlässigt wurde, sind die Konditionen für den Handel. Dazu gehört zum einen die Höhe der Rabatte für den Handel, die jedoch schwer zu messen ist. Zum anderen werden von den Verlagen Werbekostenzuschüsse bezahlt, um z. B. eine bessere Platzierung im Laden oder in den MarketingMedien des Handels zu erreichen. Doch es bleibt unklar, inwieweit diese Konditionen letztlich zu höheren Deckungsbeiträgen durch Vorteile in der Distribution führen.

\subsection{Einflussfaktoren der Preispolitik}

Die Preispolitik nimmt aufgrund der in Deutschland geltenden Buchpreisbindung eine Sonderstellung ein (Rürup/Klopfleisch/Stumpp, 1997: 2ff.). Der Verkaufspreis wird vom Verlag einmalig zum jeweiligen Einführungszeitpunkt des Buches determiniert, und im weiteren Verlauf des Lebenszyklus' gibt es eher selten zusätzliche Preisanpassungen. Auch der Handel hat kaum Möglichkeiten, im Rahmen der Preispolitik zu agieren, da Preisnachlässe nur in speziellen Ausnahmefällen (z. B. Mängelexemplare) möglich sind.

In der Vergangenheit gab es nur wenige Studien, die sich mit dem Einfluss der Preispolitik auf den Bucherfolg beschäftigt haben (Chevalier/Goolsbee, 2003; Hjorth-Andersen, 2000). Diese Forschungsfrage rückte jetzt jedoch verstärkt in den Mittelpunkt (Chevalier/Mayzlin, 2006; Clement/Proppe/Sambeth, 2006; Ghose/Smith/Telang, 2006). Ein Einflussfaktor auf den Buchabsatz ist der Verkanfspreis, der mit der Seitenanzahl hoch korreliert (Clement/Proppe/Sambeth, 2006: 12; Meckes/Krohn, 2004:3). Alle Studien kommen hier zu übereinstimmenden Ergebnissen: ein hoher Verkaufspreis wirkt sich negativ auf den Absatz des Buches aus. Ghose/Smith/Telang (2006) betrachten in diesem Zusammenhang zusätzlich die Situation des Gebrauchtbuchmarktes. Auch hier ergibt sich, dass ein hoher Preis für das gebrauchte Buch einen negativen Einfluss auf den Absatz dieses Buches hat, jedoch einen positiven Einfluss auf den Absatz des neuen Buches mit identischem Titel. 
Unberücksichtigt bleiben Informationen über den Kaufanlass (Geschenk, Eigennutzung etc.) und den Kaufgrund (Weiterbildung, Unterhaltung etc.). So kamen einige Autoren zu dem Ergebnis, dass in bestimmten Sachgebieten beim Kauf eines Buches als Geschenk eine positive Preiselastizität herrscht (z. B. van der Ploeg, 2004). In diesem Fall kann der Preis als Qualitätsindikator interpretiert werden (Völckner, 2006). Die Befunde zeigen auf, dass die Wirkungsbeziehung des Verkaufspreises nicht eindimensional ist und daher eine differenzierte Betrachtung erfordert. Auch wenn die Möglichkeiten im Rahmen der Preispolitik bisher in Deutschland stark eingeschränkt sind, so liegt die Bestimmung des anfänglichen Buchpreises in Händen der Verleger. Es ist daher von Relevanz, diese Entscheidung auf eine breite Informationsbasis zu stützen.

Im Hinblick auf die zunehmende Bedeutung des Versand-Buchhandels sind auch die Versandkosten als zusätzliche Preiskomponente zu beachten. Chevalier/Goolsbee (2003) berücksichtigen diesen Parameter explizit. Hier besteht jedoch die Problematik, dass sich der Einflussfaktor nur schwer operationalisieren lässt, da die Staffelung der Transportkosten von Online-Buchkäufen in der Regel nicht linear verläuft und zusätzlich verschiedene Versandoptionen zur Auswahl stehen. Es kann festgehalten werden, dass die Versandkosten einen negativen Einfluss auf den Online-Buchabsatz haben, auch wenn das Niveau des Einflusses schwer zu determinieren ist.

Tabelle 3 fasst die betrachteten Einflussfaktoren auf den Bucherfolg zusammen und stellt die empirisch ermittelten Wirkungsbeziehungen im Überblick dar.

\section{Implikationen für die weitere Forschung}

Aus der umfangreichen Literaturrecherche lassen sich verschiedene inbaltliche Implikationen ableiten. Zunächst wird deutlich, dass das Produktmanagement mehrfach betrachtet wurde, jedoch aufgrund der erheblichen Messprobleme auf Buchebene zentrale, subjektive Aspekte wie Cover, Thema etc. kaum analysiert wurden. Lediglich in den Leserbefragungen wird deutlich, dass diese Aspekte von Relevanz sind. Diese Ergebnisse lassen sich aber nicht unmittelbar in Handlungsempfehlungen für das Management auf Buchebene umwandeln. Festzuhalten ist jedoch, dass dem Autor (Bekanntheit und Erfahrung mit weiteren Werken) ein positiver Einfluss zuteil wird, der allerdings hinsichtlich seiner Gewinnwirkungen nicht eindeutig ist. In Bezug auf die Kommunikationspolitik werden Bestsellerlisten als erfolgsfördernd angesehen, da Titel auf der Bestsellerliste über die höhere Distributionsquote im Handel wiederum eine höhere Nachfrage generieren und so ein endogener Prozess initiiert wird, der insbesondere unbekannten Autoren hilft. Dies gilt ebenso für Rezensionen, sofern diese eindeutig positiv oder auch negativ sind - beides steigert den Erfolg besonders für unbekannte Autoren (Clement/Proppe/Sambeth, 2006). Zentral für den Buchabsatz ist ebenfalls das Vertriebsmanagement der Verlage, das überraschenderweise bislang vollkommen von der Forschung ignoriert wurde. Des Weiteren ist es von Interesse, zusätzliche Untersuchungen zur Preispolitik durchzuführen, da hier konträre Ergebnisse auftreten: positive Preiselastizität bei van der Ploeg (2004) versus negative Preiselastizität bei Clement/ Proppe/Sambeth (2006). Derartige Untersuchungen sollten auch den Kaufanlass und das Genre berücksichtigen, da dies im Zusammenhang mit dem Preis ein relevantes Kriterium zu sein scheint.

Insgesamt ist zu empfehlen, weitere Determinanten der Kaufentscheidung in die empirischen Untersuchungen einzubeziehen. Hier bieten sich insbesondere detaillierte Informationen über die Kaufgründe an, die sich an die Adoptionsforschung anlehnen sollten. Schließlich wäre auch ein Vergleich der Ergebnisse im internationalen Kontext 
Tabelle 3: Zusammenfassung der Einflussfaktoren des Marketing-Mix

\begin{tabular}{|c|c|c|c|c|c|c|c|}
\hline \multicolumn{8}{|c|}{ Elemente des Marketing-Mix } \\
\hline Produkt & & $\begin{array}{l}\text { Kommunika- } \\
\text { tion }\end{array}$ & & Distribution & & Preis & \\
\hline $\begin{array}{l}\text { Autor } \\
\text { - Bekanntheit } \\
\text { - Person } \\
\text { - Herkunft } \\
\text { - Biografien }\end{array}$ & $\begin{array}{l}++ \\
. \\
. \\
.\end{array}$ & $\begin{array}{l}\text { Werbung } \\
\text { - für Kunden } \\
\text { - für Handel } \\
\text { - für Kritiker }\end{array}$ & $\begin{array}{l}+/ \text { n.s. } \\
. / \\
. /\end{array}$ & $\begin{array}{l}\text { Verlag } \\
\text { - Größe } \\
\text { - Image }\end{array}$ & $\begin{array}{l}+/ \text { n.s. } \\
+\end{array}$ & $\begin{array}{l}\text { Verkaufs- } \\
\text { preis } \\
\text { - neues Buch } \\
\text { - Gebraucht- } \\
\text { buch }\end{array}$ & $\begin{array}{l} \\
- \\
+ \\
+\end{array}$ \\
\hline $\begin{array}{l}\text { Inhalt } \\
\text { - Aktualität } \\
\text { - Thema } \\
\end{array}$ & $\begin{array}{l}+/ \text { n.s. } \\
. /\end{array}$ & $\begin{array}{l}\text { Rezensionen } \\
\text { - durch Kritiker } \\
\text { - durch Kunden }\end{array}$ & $\begin{array}{l}+/ \text { n.s. } \\
+ \\
\end{array}$ & $\begin{array}{l}\text { Timing } \\
\text { - Editionsform } \\
\text { - saisonal } \\
\end{array}$ & $\begin{array}{l}. \\
. \\
.\end{array}$ & $\begin{array}{l}\text { Versandkos- } \\
\text { ten }\end{array}$ & $(-)$ \\
\hline $\begin{array}{l}\text { Beschreibung } \\
\text { - Titel } \\
\text { - Sachgebiet/ } \\
\text { Genre } \\
\text { - Klappentext } \\
\end{array}$ & $\begin{array}{l}. / \\
. \\
.\end{array}$ & $\begin{array}{l}\text { Ratings } \\
\text { - Bestsellerlisten } \\
\text { - Preise/Aus- } \\
\text { zeichnungen }\end{array}$ & $\begin{array}{l}+ \\
++ \\
(+)\end{array}$ & Vertriebskanal &. & & \\
\hline $\begin{array}{l}\text { Erscheinungsbild } \\
\text { - Editionsform } \\
\text { - Cover } \\
\text { - Sprache } \\
\text { - Umfang } \\
\end{array}$ & $\begin{array}{l}+/- \\
+ \\
. / \\
. /\end{array}$ & $\begin{array}{l}\text { Mund-Propa- } \\
\text { ganda }\end{array}$ & $(+)$ & Versanddauer & $(-)$ & & \\
\hline $\begin{array}{l}\text { Cross-Selling } \\
\text { - Komplemen- } \\
\text { tärgüter } \\
\text { - Serien } \\
\end{array}$ & $\begin{array}{l}+ \\
\text { n.s. }\end{array}$ & & & $\begin{array}{l}\text { Rabatt für den } \\
\text { Handel }\end{array}$ &. & & \\
\hline
\end{tabular}

wünschenswert, denn es dominieren empirische Studien aus den USA und den Niederlanden.

Auch aus methodischer Sicht lassen sich mehrere Forschungsimplikationen festhalten: Zunächst wird deutlich, dass sich der Großteil der Studien auf einige wenige Einflussfaktoren beschränkt. Dies ist umso kritischer, da die (Regressions-)Ergebnisse durch eine Nicht-Berücksichtigung von zentralen Variablen verzerrt sind. Allerdings sind bei hedonischen Gütern viele relevante Variablen (z. B. das Thema oder Cover eines Buches) nur schwierig zu messen. Dennoch existieren zahlreiche Methoden (z. B. die Inhaltsanalyse), die für diesen Forschungszweig zentrale Einflüsse - wenngleich auch subjektiv - messbar machen können.

Die Studien verwenden zudem sehr unterschiedliche Operationalisierungen der Einflussgrößen und - zumindest teilweise - unzureichende Modellierungen, so dass die Vergleichbarkeit der Ergebnisse nicht unmittelbar gegeben ist. Dies gilt umso mehr, wenn auch das Erfolgsmaß variiert. Hierbei ist vor allem nach einer gewinnorientierten Messung des Erfolgs zu streben. Dies ist allerdings in der Praxis keineswegs trivial, denn selbst für die Verlage ist es eher schwierig, über den Deckungsbeitrag hinaus die Vollkosten auf Buchebene verursachungsbedingt zu ermitteln, so dass der Absatz derzeit noch das beste Erfolgsmaß darstellt.

Allgemein betrachtet ist für die weitere Erfolgsfaktorenforschung im Buchbereich 
ein Ausgleich der bisherigen Defizite wünschenswert. Das Forschungsfeld steht sowohl in Bezug auf Innovationen allgemein als auch im Hinblick auf vergleichbare hedonische Güter (Spielfilme, Musik etc.) noch am Anfang. Ebenso werden Neuheiten im Bereich der neuen Medien bisher nur sehr selten berücksichtigt (Hörbücher, Books-on-Demand etc.). Hier ergeben sich vielfältige und neue Forschungsfelder, die wirtschaftlich bereits von hoher Relevanz sind.

\section{Literatur}

Basuroy, Suman / Chatterjee, Subimal / Ravid, Abraham S. (2003): How Critical are Critical Reviews? The Box Office Influence of Film Critics, Star-Power, and Budgets. In: Journal of Marketing, 67 (October), S. 103-117.

Baumgarth, Carsten (2004): Erfolgreiche Führung von Medienmarken: Strategien für Positionierung, Markentransfers und Branding, Wiesbaden.

Beck, Jonathan (2007): The sales effect of word of mouth: a model for creative goods and estimates for novels. In: Journal of Cultural Economics, 31 (1), S. 5-23.

Bonfadelli, Heinz (2004): Medienwirkungsforschung - Grundlagen, Band 1, Konstanz.

Bonfadelli, Heinz / Fritz, Angela (1993): Lesen im Alltag von Jugendlichen. In: BertelsmannStiftung (Hrsg.): Lesesozialisation - Leseerfahrungen und Lesekarrieren, Band 2, Gütersloh, S. 7-214.

Börsenverein des deutschen Buchhandels (2005a): Buch und Buchhandel in Zahlen 2005, Frankfurt am Main.

Börsenverein des deutschen Buchhandels (2005b): Buchkäufer und Leser 2005 - Profile, Motive, Wünsche, Studienreihe Marktforschung - Kurzfassung, Frankfurt am Main.

Börsenverein des deutschen Buchhandels (2005c): Der Belletristikmarkt 2004 - Bedeutung und Stellenwert von Sonderausgaben, Präsentation vom 17.03.2005, Frankfurt am Main.

Börsenverein des deutschen Buchhandels (2006a): Buch und Buchhandel in Zahlen 2006, Frankfurt am Main.

Börsenverein des deutschen Buchhandels (2006b): Der Markt der Billigbücher - Ergebnisse der GfK-Studie 2006, Präsentation des Referats Marketing und Marktforschung / Verleger-Ausschuss, Frankfurt am Main.

Börsenverein des deutschen Buchhandels (2006c): Buchhandlungen und Neue Medien - Chancen, Visionen und Handlungskonzepte für den stationären Buchhandel aus Sicht strategischer Zielgruppen, Referat Marketing und Marktforschung, Frankfurt am Main.

Börsenverein des deutschen Buchhandels (2007): Buch und Buchhandel in Zahlen 2007, Frankfurt am Main.

Bradlow, Eric T. / Fader, Peter S. (2001): A Bayesian Lifetime Model for the „Hot 100“ Billboard Songs. In: Journal of the American Statistical Association, 96 (454), S. 369-381.

Brockhoff, Klaus (1993): Produktpolitik, 3. Auflage, Stuttgart.

Brown, Stephen (2001): Marketing for Muggles: Harry Potter and the Retro Revolution. In: Journal of Marketing Management, 17 (5/6), S. 463-479.

Chandon, Pierre / Morwitz, Vicki G. / Reinartz, Werner J. (2005): Do Intentions Really Predict Behavior? Self-Generated Validity Effects in Survey Research. In: Journal of Marketing, 69 (April), S. 1-14.

Chevalier, Judith A. / Mayzlin, Dina (2006): The Effect of Word of Mouth on Sales: Online Book Reviews. In: Journal of Marketing Research, 43 (3), S. 345-354.

Chevalier, Judith A. / Goolsbee, Austan (2003): Measuring Prices and Price Competition Online: Amazon.com and BarnesandNoble.com. In: Quantitative Marketing and Economics, 1 (2), S. 203-222.

Clement, Michel (2004): Erfolgsfaktoren von Spielfilmen im Kino - Eine Übersicht der empirischen betriebswirtschaftlichen Literatur. In: Medien E Kommunikationswissenschaft, 52 (2), S. 250-271. 
Clement, Michel / Proppe, Dennis / Sambeth, Frank (2006): Der Einfluss von Meinungsführern auf den Erfolg von hedonischen Produkten. In: Zeitschrift für Betriebswirtschaft, 76 (7/8), S. 1-28.

Clement, Michel / Sambeth, Frank (2004): Buchkritiker und Bucherfolg: Wie ist der Einfluss wirklich? In: Medienwirtschaft, 3 (1), S. 105-114.

Clerides, Sofronis K. (2002): Book Value: Intertemporal Pricing and Quality Discrimination in the US Market for Books. In: International Journal of Industrial Organization, 20 (10), S. $1385-1408$.

Czepek, Andrea (2001): Star-Enthüllungen spülen mehr Geld in die Kassen. In: Buchreport/Magazin, (Dezember), S. 89-91.

D’Astous, Alain / Colbert, François / Mbarek, Imene (2006): Factors influencing readers' interest in new book releases: An experimental study. In: Poetics, 34 (2), S. 134-147.

Dhar, Ravi / Wertenbroch, Klaus (2000): Consumer Choice Between Hedonic and Utilitarian Goods. In: Journal of Marketing Research, 37 (2), S. 60-71.

Dijkstra, Katinka (1994): Leseentscheidung und Lektürewahl: Eine empirische Untersuchung über Einflußfaktoren auf das Leseverhalten, Berlin.

Ebbinghaus, Uwe (2003): Frisch verkostet. In: Börsenblatt, 170 (30), S. 13-15.

Eliashberg, Jehoshua / Elberse, Anita / Leenders, Mark A. A. M. (2006): The Motion Picture Industry: Critical Issues in Practice, Current Research, and New Research Directions, Marketing Science, 25 (6), S. 638-661.

Faulstich, Werner (1983): Bestandsaufnahme Bestseller-Forschung, Wiesbaden.

Fritz, Angela (1989): Lesen in der Mediengesellschaft: Standortbeschreibung einer Kulturtechnik, Wien.

Ghose, Anindya / Smith, Michael D. / Telang, Rahul (2006): Internet Exchanges for Used Books: An Empirical Analysis of Product Cannibalization and Welfare Impact. In: Information Systems Research, 17 (1), S. 3-19.

Harmgarth, Friederike (1997): Lesegewohnheiten - Lesebarrieren: öffentliche Bibliothek und Schule - neue Formen der Partnerschaft, Gütersloh.

Harmgarth, Friederike (Hrsg.) (1999): Das Lesebarometer, Gütersloh.

Hauschildt, Jürgen (2004): Innovationsmanagement, 3. Auflage, München.

Hirschman, Elizabeth C. / Holbrook, Morris B. (1982): Hedonic Consumption: Emerging Concepts, Methods and Propositions. In: Journal of Marketing, 46 (Summer), S. 92-101.

Hjorth-Andersen, Christian (2000): A Model of the Danish Book Market. In: Journal of Cultural Economics, 24 (1), S. 27-43.

Hoffmann, Hilmar (1994): Der Buch-Film-Hit. Das neue Verhältnis von Literatur und Film. In: Brinkemper, Peter V. / von Dadelsen, Bernhard / Seng, Thomas (Hrsg.): World Media Park, Berlin, S. 108-119.

Innofact AG (2006): Verlagsranking 2006, Düsseldorf.

Janssen, Susanne / Leemans, Hein (1988): Differences in Consumer Behavior between Buyers of Literature. In: Poetics, 17 (6), S. 563-575.

Kamphuis, Jan (1991): Satisfaction with books: Some empirical findings. In: Poetics, 20 (5-6), S. $471-485$.

Köcher, Renate (1988): Familie und Lesen. Eine Untersuchung über den Einfluß des Elternhauses auf das Leseverhalten. In: Archiv für Soziologie und Wirtschaftsfragen des Buchbandels, 63, S. W2275-W2364.

Köcher, Renate (1993): Lesekarrieren - Kontinuität und Brüche. In: Bertelsmann-Stiftung (Hrsg.): Lesesozialisation - Leseerfahrungen und Lesekarrieren, Band 2, Gütersloh, S. 215-310.

Lageat, Thierry / Czellar, Sandor / Laurent, Gilles (2003): Engineering Hedonic Attributes to Generate Perceptions of Luxury: Consumer Perception of an Everyday Sound. In: Marketing Letters, 14 (2), S. 97-109.

Lauterbach, Burkhart R. (1979): Bestseller: Produktions- und Verkaufsstrategien, Tübingen.

Lee, Jonathan / Boatwright, Peter / Kamakura, Wagner A. (2003): A Bayesian Model for Prelaunch Sales Forecasting of Recorded Music. In: Management Science, 49 (2), S. 179-196.

Leemans, Hein / Stokmans, Mia J. W. (1991): Attributes Used in Choosing Books. In: Poetics, 20 (5), S. 487-505. 
Lehmann, Donald R. / Weinberg, Charles B. (2000): Sales through Sequential Distribution Channels: An Application to Movies and Videos. In: Journal of Marketing, 64 (3), S. 18-33.

Lenz, Daniel (2003): Unverwechselbarkeit erstickt im Einheitsbrei. In: Buchreport/Magazin, 34 (August), S. 9-13.

Liebenstein, Karina (2005): Bestsellerlisten 1962-2001. Eine statistische Analyse. In: Rautenberg, Ursula / Titel, Volker (Hrsg.): Alles Buch. Studien der Erlanger Buchwissenschaft XII, Erlangen-Nürnberg.

Link, Henry C. / Hopf, Harry A. (1946): People and Books. A Study of Reading and Book-buying Habits, New York.

Ludes, Peter (1989): Stars in soziologischer Perspektive. In: Thomsen, Christian W. / Faulstich, Werner (Hrsg.): Seller, Stars und Serien: Medien im Produktverbund, Heidelberg, S. 213-265.

Martin, Eva / Scheer, Thomas (1995): Erfolgsfaktor „Zufriedene Kunden“. In: Kind, Hero (Hrsg.): Buchmarketing, Düsseldorf et al., S. 27-57.

Maskos, Rebecca (2006): Bücher sind Nebensache - Verleger entdecken das MerchandisingGeschäft. In: Radiofeuilleton, http://www.dradio.de/dkultur/sendungen/thema/573587/, 13.12.2006

McQuivey, James L. / McQuivey, Megan K. (1998): Is It a Small Publishing World After All?: Media Monopolization of the Children's Book Market, 1992-1995. In: Journal of Media Economics, 11 (4), S. 35-48.

Meckes, Rainer / Krohn, Felix (2004): Ertragssteigerung für Buchverlage - Mit optimierter Preissetzung aus der Krise, Simon Kucher \& Partner, Bonn et al.

Miller, Christopher M. / McIntyre, Shelby H. / Mantrala, Murali K. (1993): Toward Formalizing Fashion Theory. In: Journal of Marketing Research, 30 (2), S. 142-157.

Noelle-Neumann, Elisabeth / Schulz, Rüdiger (1987): Typologie der Käufer und Leser. In: Archiv für Soziologie und Wirtschaftsfragen des Buchhandels, Beilage zum Börsenblatt des Deutschen Buchbandels, 62 (1), S. W2163-2256.

o. V. (2003): 1.713 Buchkäufer beurteilen Buchverlage. In: BuchMarkt, 38 (September), S. 36-37.

Piters, Ronald A. M. P. / Stokmans, Mia J. W. (2000): Genre Categorization and its Effect on Preference for Fiction Books. In: Empirical Studies of the Arts, 18 (2), S. 159-166.

Prosi, Gerhard (1971): Ökonomische Theorie des Buches, Düsseldorf.

Radas, Sonja / Shugan, Steven M. (1998): Seasonal Marketing and Timing New Product Introductions. In: Journal of Marketing Research, 35 (3), S. 296-315.

Reibstein, David J. / Farris, Paul W. (1995): Market Share and Distribution: A Generalization, a Speculation, and some Implications. In: Marketing Science, 14 (3), S. G190-G202.

Rürup, Bert / Klopfleisch, Roland / Stumpp, Henning (1997): Ökonomische Analyse der Buchpreisbindung, Frankfurt am Main.

Saxer, Ulrich / Langenbucher, Wolfgang / Fritz, Angela (1989): Kommunikationsverhalten und Medien, Gütersloh.

Schenkel, Hubertus (1995): In Vorbereitung auf das Jahr 2000. Herausforderungen an das Verlagsmarketing. In: Kind, Hero (Hrsg.): Buchmarketing, Düsseldorf et al., S. 17-25.

Schmidtchen, Gerhard (1974): Lesekultur in Deutschland. In: Archiv für Soziologie und Wirtschaftsfragen des Buchhandels, Beilage zum Börsenblatt des Deutschen Buchbandels, 30, S. 707-896.

Schweitzer, Pascal (1997): Der Buchmarkt in Frankreich: Struktur, Organisation, Zahlen, Friedrichsdorf.

Sieg, Anja (2001): In den US-Verlagen steht Black für Erfolg. In: Buchreport/Magazin, S. 37-38.

Sieg, Anja (2003): US-Buchmarkt baut auf Minderheit mit Kaufkraft. In: Buchreport/Magazin, 34, S. 36-37.

Smith, Michael D. / Brynjolfsson, Erik (2001): Consumer Decision-Making at an Internet Shopbot: Brand Still Matters. In: Journal of Industrial Economics, 49 (4), S. 541-558.

Sorensen, Alan T. (2004): Bestseller Lists and Product Variety: The Case of Book Sales, Working Paper at Stanford University, erscheint demnächst in: Journal of Industrial Economics.

Sorensen, Alan T. / Rasmussen, Scott J. (2004): Is Any Publicity Good Publicity? A Note on the Impact of Book Reviews, Working Paper at Stanford University.

Stiftung Lesen (1993): Leseverhalten in Deutschland 1992/93, Mainz. 
Stockem, Anno (1988): Vermarktung von Büchern, Wiesbaden.

Stokmans, Mia J. W. (2002): Do readers agree on the genre categories of literary fiction? In: Latheenmaki, Satu (Hrsg.): Stability and Dynamics of Power - 16. Annual Colloquium on Research in Economic Psychology, Working Paper at Turku School of Economics and Business Administration, S. 165-170.

Stokmans, Mia J. W. / Hendrickx, M. (1994): The attention paid to new book releases on a display table. In: Poetics, 22 (3), S. 185-197.

Tietzel, Manfred (1992): Goethe - ein Homo oeconomicus. In: Tietzel, Manfred (Hrsg.): Kunst und Ökonomie, München, S. 303-355.

Tietzel, Manfred (1995): Literaturökonomik, Tübingen.

van der Ploeg, Frederick (2004): Beyond the Dogma of the Fixed Book Price Agreement. In: Journal of Cultural Economics, 28, S. 1-20.

van Eimeren, Birgit / Ridder, Christa-Maria (2005): Trends in der Nutzung und Bewertung der Medien 1970 bis 2005. Ergebnisse der ARD/ZDF Langzeitstudie Massenkommunikation. In: Media Perspektiven, 10, S. 490-504.

Völckner, Franziska (2006): Determinanten der Informationsfunktion des Preises: Eine empirische Analyse. In: Zeitschrift für Betriebswirtschaft (ZfB), 76 (5), S. 473-497.

Vogel, Harold L. (2004): Entertainment Industry Economics, Cambridge.

Wedel, Michel / Kamakura, Wagner A. (2001): Market Segmentation: Conceptual and Methodological Foundations, Boston, Mass.

Weinhold, Heinz (1956): Marktforschung für das Buch, St. Gallen.

Wilke, Jürgen / König, Barbara (1997): Hilft das Fernsehen der Literatur? Auch eine Antwort auf die Preisfrage der Deutschen Akademie für Sprache und Dichtung. In: Füssel, Stephan (Hrsg.): Gutenberg Jahrbuch 1997, Mainz, S. 245-282.

Wirtz, Bernd W. (2000): Medien- und Internetmanagement, Wiesbaden.

Zentes, Joachim / Effen, Ingrid (1995): Perspektiven für den Buchmarkt, Düsseldorf.

Ziermann, Klaus (2000): Der deutsche Buch- und Taschenbuchmarkt 1945-1995, Berlin. 